\title{
Eighth International Symposium on Intensive Care and Emergency Medicine for Latin America
}

\author{
Sao Paolo, Brazil. 17-20 June 2015 \\ Published: 28 September 2015 \\ These abstracts are available online at http://www.ccforum.com/supplements/19/s2
}

\section{POSTER PRESENTATIONS}

\section{P1}

Indirect calorimetry in the pediatric ICU

Zina Maria Almeida de Azevedo ${ }^{1}$, Daniella B Moore ${ }^{1 *}$

Luis Fernando P Amendola², Margarida dos Santos Salú ${ }^{2}$, Daniella ML Caixeta², Elza Rosa Pedroso², Djamayna VC Oliveira², Eloane Gonçalves Ramos ${ }^{2}$

${ }^{1}$ Universidade Federal Fluminense, Flamengo, Rio de Janeiro, São Paulo, São Paulo, Brazil; ' Instituto Fernandes Figueira-Fiocruz, Flamengo, Rio de Janeiro, São Paulo, São Paulo, Brazil

Critical Care 2015, 19(Suppl 2):P1; doi:10.1186/cc14659

Introduction: Failure to accurately estimate energy requirements may result in an impaired recovery. Overfeeding has been associated with increased carbon dioxide production, respiratory failure, hyperglycemia and fat deposits in the liver, while underfeeding can lead to malnutrition, muscle weakness and impaired immunity.

Objective: This study aimed to determine the metabolic profile of infant and preschool children submitted to mechanical ventilation in the ICU.

Methods: A prospective study was carried out in a pediatric ICU in Rio de Janeiro that included children aged from 1 month to 6 years submitted to mechanical ventilation from June 2013 to May 2015. Indirect calorimetry was used to obtain resting energy expenditure (REE) and oxygen consumption $\left(\mathrm{VO}_{2}\right)$ in the first 48 hours of admission. The predicted basal metabolic rate (PBMR) was calculated using the Schofield equation. The metabolic state of each patient was assigned as hypermetabolic (REE/PBMR $>110 \%$ ), hypometabolic (REE/PBMR $<90 \%$ ) or normal (REE/PBMR 90-110\%). The ratio of caloric intake to REE was also calculated and ratios of $>1.5$ and $<0.5$ were classified as overfeeding and underfeeding respectively.

Results: A total of 35 infants and 17 preschool children were included. The male/female ratio was 34/18. In respect of severity of sepsis, 19 patients had septic shock, 24 had sepsis, five had severe sepsis and four had systemic inflammatory response syndrome. We observed a high incidence of hypometabolism (88.5\%) and a low incidence of normal metabolism $(7.7 \%)$ and hypermetabolism (3.8\%). A low value of $\mathrm{VO}_{2}$ was observed in $46.1 \%$ of the patients $\left(\mathrm{VO}_{2} \leq 120 \mathrm{ml} /\right.$ minute $\left./ \mathrm{m}^{2}\right)$, a normal value in $40.4 \%$ $\left(\mathrm{VO}_{2}>120\right.$ to $\left.\leq 160 \mathrm{ml} / \mathrm{minute} / \mathrm{m}^{2}\right)$ and a high value in only $13.5 \%$ of the patients $\left(\mathrm{VO}_{2}>160 \mathrm{ml} / \mathrm{minute} / \mathrm{m}^{2}\right)$. Among the 52 included patients, 18 were fasting at the moment of the examination. The ratio of caloric intake to REE for the remaining 34 patients showed $38.2 \%$ overfeeding, $11.8 \%$ underfeeding and $50.0 \%$ normal feeding.

Conclusion: Predictive equations do not accurately predict REE in critically ill infants and preschool children, resulting in inadequate feeding. Although hypermetabolism and enhanced energy expenditure are the main clinical features of critical illness in adults, the majority of our patients were found to be hypometabolic which reinforces the need for a different approach between adult and pediatric critically ill patients.

Acknowledgements: This study was supported by FIOCRUZ-Fernandes Figueira Institute (PIP: IFF-008-FIO-13-3-13).
P4

Endothelial glycocalyx damage in patients with acute coronary syndrome

Carlos H Miranda*, Andre Schmidt, Antônio P Filho, Marcos C Borges Division of Emergency Medicine, Ribeirão Preto School of Medicine, São

Paulo University, Ribeirão Preto, São Paulo, Brazil

Critical Care 2015, 19(Suppl 2):P4; doi:10.1186/cc14660

Introduction: The endothelial glycocalyx is a thin layer of proteinaceous material at the endothelial surface of the vessels. Damage to this structure can cause protein extravasation and tissue edema, increase platelet and leukocyte adhesion and increase of the shear stress in the vessel due to decreasing the nitric oxide production. On the other hand, a vulnerable atherosclerotic plaque with rupture or erosion characterizes the acute coronary syndrome (ACS). The glycocalyx damage could contribute to this process.

Objective: To evaluate the glycocalyx damage through syndecan-1 dosage in patients with ACS.

Methods: We included patients with ACS diagnosis $(n=140)$. These patients had ST-segment elevation myocardial infarction $(n=71)$, were without ST-segment elevation myocardial infarction $(n=58)$ and had unstable angina $(n=12)$. The syndecan-1 level was dosage through commercial ELISA kits (Abcam ${ }^{\oplus}$, Cambridge, UK) at admission. These levels were compared with 45 patients with noncoronary chest pain (NCCP) in which the ACS diagnosis was ruled out through negative electrocardiogram, troponin and imaging test (tomographic or conventional coronary angiography, stress echocardiography) and 24 completely healthy individuals (CONTROL). The values were expressed in median plus percentiles $25\left(\mathrm{P}_{25}\right)$ and $75\left(\mathrm{P}_{75}\right)$.

Results: The syndecan-1 levels were higher in the patients with ACS (77 ng/ml; 46-134) compared with the NCCP group (60 ng/ml; 32-79); $p=0.01$ and CONTROL group (42 ng/ml; 27-80); $p=0.001$. No difference was observed between the NCCP versus CONTROL group ( $p=0.83$ ). A syndecan-1 level higher than $148 \mathrm{ng} / \mathrm{ml}$ was associated with the ACS diagnosis (relative risk: 1.53; $95 \%$ confidence interval: 1.33-1.80; $p<0.0001)$.

Conclusion: The endothelial glycocalyx damage could contribute to the atherosclerotic plaque vulnerability and the establishment of an ACS.

P5

Factors related to pain after cardiac surgery

Ana Elza O Mendonça*, Gilson V Torres, Luciana A Reis, Marina G Salvetti, Thaiza TX Nobre

Universidade Federal do Rio Grande do Norte, Lagoa Nova, Natal, Rio Grande do Norte, Brazil

Critical Care 2015, 19(Suppl 2):P5; doi:10.1186/cc14661

Introduction: Pain related to heart surgery is a limiting factor for patient recovery and it is moderate to severe in $40-60 \%$ of patients. 
Objective: To analyze the risk factors for the presence of pain in patients after cardiac surgery.

Methods: A prospective study in two hospitals in the city of Natal, RN. The sample consisted of 160 patients undergoing cardiac surgery, $57.5 \%$ male, mean age 56.8 years $(S D=14.4)$. Pain was evaluated by the numerical pain rating scale between days 1 and 5 after surgery. The variables that present $p<0.20$ in the bivariate analysis were selected for multivariate analysis by logistic regression to establish a pain model prediction.

Results: The factors associated with pain in the bivariate analysis were gender, age, race, work, diabetes, obesity, back pain, cardiopulmonary bypass, mediastinal drain, side and mediastinal drain, anesthesia, surgical time, coughing, vomiting, drain time and medication. Multivariate analysis allowed the identification of six risk factors for the occurrence of pain $(p<0.05)$ : surgery lasting more than three hours, side and mediastinal drain, cough, vomiting, drain time for more than 24 hours and being female.

Conclusion: This study identified risk factors for pain related to heart surgery. Knowing these factors allows enhance care planning in order to prevent and minimize the occurrence of pain in the postoperative period.

\section{P6}

Heart rate variability in HIV-infected and AIDS

Gabriela AM Galdino*, Fernanda R Moraes, Gesileny V Silva, Marilita F Accioly Universidade Federal do Triângulo Mineiro, Av. Getúlio Guaritá S/N, Abadia, Uberaba, Minas Gerais, Brazil

Critical Care 2015, 19(Suppl 2):P6; doi:10.1186/cc14662

Introduction: The availability of antiretroviral therapy (ART) has resulted in a dramatic reduction in HIV-associated mortality [1]. However, it is also known to induce an array of adverse effects [2-4], as autonomic dysfunction. The use of heart rate variability (HRV), allows the assessment of relative contribution of sympathetic and parasympathetic involvement [1].

Objective: The objective of this study was to assess the presence of autonomic dysfunction in HIV-infected and acquired immunodeficiency syndrome (AIDS) patients that receive ART. In order to do so, analysis of HRV was applied.

Methods: A total of 15 subjects with HIV and 34 with AIDS were analyzed. To capture HRV, the heart rate monitor Polar RS810CX was used. The HRV was recorded in a supine position for 20 minutes, and then while performing the respiratory sinus arrhythmia (RSA). The statistical analysis was calculated using ANOVA and Pearson correlation $(\alpha=5 \%)$.

Results: The HIV group had average duration of exposure to the virus of $4.5 \pm 3.5$ years, and the AIDS group $12.6 \pm 6.6$ years, both under ART. Only the \%REC presented suggestive statistically significant difference (Table 1) of greater parasympathetic modulation in the AIDS group, while the RSA did not show a statistically significant difference $(p>0.05)$. Conclusion: Based on chaos theory, \%REC was more sensitive to identify lower parasympathetic modulation in the HIV group, probably due to shorter exposure to antiretroviral therapy. Therefore, it is suggested that the medication used by the population modifies the HRV.

Acknowledgements: Supported by FAPEMIG

References

1. Lebech AM, Kristoffersen US, Mehlsen J, Wiinberg N, Petersen CL, Hesse B, et al: Autonomic dysfunction in HIV patients on antiretroviral therapy: studies of heart rate variability. Clin Physiol Funct Imaging 2007, 27(6):363-367.

2. Carr A, Samaras K, Burton S, Law M, Freund J, Chisholm DJ, Cooper DA: A syndrome of peripheral lipodystrophy, hyperlipidaemia and insulin resistance in patients receiving HIV protease inhibitors. AIDS 1998, 12(7) F51-F58.

Table 1(abstract cc14662) Time domain analysis

\begin{tabular}{llll}
\hline HRV parameter (ms/\%) & HIV & AIDS & $\boldsymbol{p}$ value \\
\hline RMSSD & $18.5(11.5)$ & $23.7(17)$ & $<0.314$ \\
PNN50 & $3.9(6.8)$ & $6.9(9.9)$ & $<0.340$ \\
\%REC & $38.3(11.2)$ & $32.7(6.7)$ & $<0.002$ \\
\hline
\end{tabular}

Data presented as mean (SD)
3. Dube MP, Sattler FR: Metabolic complications of antiretroviral therapies. AIDS Clin Care 1998, 10(6):41-44

4. Ramirez-Villegas JF, Lam-Espinosa E, Ramirez-Moreno DF, CalvoEcheverry PC, AgredoRodriguez W: Heart rate variability dynamics for the prognosis of. cardiovascular risk. PLoS One 2011, 6(2):e17060.

P7 Impact of chest pain protocol in the use of fibrinolytic therapy in a private hospital network with access to telemedicine in a middle income country

Pedro Gabriel MB Silva*, Antonio Baruzzi, Giuliano Generoso, Henrique Ribeiro, Jose Carlos Teixeira, Marcelo Jamus, Mariana Y Okada, Sheila Simoes,

Thiago A Macedo, Valter Furlan

Hospital Totalcor, Cerqueira Cesar, São Paulo, São Paulo, Brazil

Critical Care 2015, 19(Suppl 2):P7; doi:10.1186/cc14663

Introduction: Brazilian registries [1] have shown that there is a gap between evidence-based therapies and the real treatment provided to patients with myocardial infarction. A chest pain protocol was implemented in a private hospital group in 2012 aiming at standardized optimal care for these patients.

Objective: To evaluate the hypothesis of improving the use of reperfusion therapy and benefit in clinical outcomes in patients with STEMI after 2 years of implementation of the protocol in a large chest pain network. Methods: In 2012, physicians and nurses from 22 emergencies were trained to comply with a chest pain protocol and were provided access to telemedicine with a reference cardiologist available 24 hours a day, 7 days a week, for clinical discussion. All cases of ST segment elevation myocardial infarction (STEMI) were transferred to a reference hospital and the use of fibrinolytics before transfer (pharmacoinvasive strategy) was recommended. Data of STEMI patients transferred in 2011 (before protocol and telemedicine) were compared with the patients treated in 2013/14 (after implementation). A maximum limit of significance of 5\% was defined for the chance of type I error $(p<0.05$ was considered statistically significant).

Results: The number of patients transferred to the reference hospital was 113 (2011), 140 (2013) and 123 (2014). Fibrinolytic therapy was used in 43 patients (38\%) in 2011 and in 147 cases $(55.8 \%)$ in 2013/14 ( $p=$ 0.002 ). The mortality rates of transferred STEMI cases were $8 \%$ in 2011 (nine cases) and 3\% (eight cases) in 2013/14 $(p=0.06)$ with an observed/ expected mortality by Grace score of $1.11(95 \% \mathrm{Cl}, 0.39-1.83)$ in 2011 and $0.68(95 \% \mathrm{Cl}, 0.21-1.15)$ in 2013/14. Along the years of 2011, 2012 and 2013 , two patients treated with fibrinolytics died during the hospital stay $(1.05 \%)$, whereas hospital mortality was $8.06 \%$ among those not treated with thrombolysis $(p<0.001)$. The patients that received reperfusion therapy in the first hospital used telemedicine more frequently $(63.3 \%$ versus $42.2 \% ; p=0.001$ ). See Table 1 .

Conclusion: Two years after implementation of a chest pain protocol in a private emergency network, there was significant increase in the use of reperfusion therapy probably explained by a more frequent use of telemedicine in the group treated with reperfusion therapy.

Table 1(abstract cc14663) Baseline characteristics

\begin{tabular}{llll}
\hline & $\mathbf{2 0 1 1}(\boldsymbol{n}=$ & $\mathbf{2 0 1 3 / 1 4}(\boldsymbol{n}=$ & $\boldsymbol{p}$ \\
& $\mathbf{1 1 3})$ & $\mathbf{2 6 3})$ & value \\
\hline Mean age (years) & $59.6( \pm 13)$ & $58.7( \pm 11)$ & 0.49 \\
Male & $70 \%$ & $74 \%$ & 0.47 \\
Hypertension & $62 \%$ & $64 \%$ & 0.81 \\
Diabetes & $26 \%$ & $29 \%$ & 0.61 \\
Previous PCI or CABG & $14.2 \%$ & $10.6 \%$ & 0.43 \\
$\begin{array}{l}\text { Previous myocardial } \\
\text { infarction }\end{array}$ & $7.1 \%$ & $5.7 \%$ & 0.78 \\
$\begin{array}{l}\text { Mean Grace risk score } \\
\text { (mortality) }\end{array}$ & $7.2 \%$ & $4.4 \%$ & 0.5 \\
\hline PCI percutan & & & \\
\hline
\end{tabular}

$\mathrm{PCl}$ percutaneous coronary intervention, $C A B G$ coronary artery bypass graft 


\section{Reference}

1. Nicolau JC, Franken M, Lotufo PA, Carvalho AC, Marin Neto JA, Lima FG, et al: Utilização de terapêuticas comprovadamente úteis no tratamento da coronariopatia aguda:comparação entre diferentes regiões brasileiras. Análise do Registro Brasileiro de Síndromes Coronarianas Agudas (BRACE-Brazilian Registry on Acute Coronary Syndromes). Arq Bras Cardiol 2012, 98(4):282-289.

P8

Implementation and certification of a heart failure clinical care program in a middle income country: impact in clinical outcomes after $\mathbf{2}$ years

Pedro Gabriel MB Silva*, Antonio Baruzzi, Douglas Ribeiro, Flavio Brito, Giuliano Generoso, Jose Teixeira, Marcelo Jamus, Mariana Okada,

Thiago Macedo, Valter Furlan

Hospital Totalcor, Cerqueira Cesar, São Paulo, São Paulo, Brazil

Critical Care 2015, 19(Suppl 2):P8; doi:10.1186/cc14664

Introduction: Clinical care programs (CCP) that monitor and optimize care have the potential to improve outcomes; however, their real benefits are still controversial.

Objective: This study aims to evaluate the hypothesis of benefits in clinical outcomes after 2 years of a CCP.

Methods: Prospective study of consecutive patients hospitalized with HF in a Brazilian private cardiovascular center. Two groups were compared based on the time to CCP initiation: the historical group, compounded by patients from the 6 months prior to CCP (group 1); and the intervention group, compounded by patients admitted with diagnosis of HF from July 2012 until June 2014, the period when patients and staff were monitored on a daily basis by a case manager nurse and a medical leader which provided educational interventions. The CCP was certified by an international society in October 2012.

Results: In a total of 2188 patients, the mean age was 69.3 years and $55.8 \%$ were male (Table 1). Evidence-based therapies at hospital discharge (ACEI/ARB and beta-blocker in eligible patients) showed no significant change (95.8\% pre-CCP and $97.5 \%$ post-CCP; $p=0.12$ ). The outcomes analyzed in groups 1 and 2, were, respectively: hospital readmissions due to $\mathrm{HF}$ within 30 days $(13.9 \%$ vs. $9.1 \% ; p=0.008)$; length of stay ( $8.9 \pm 7.9$ days vs. $7.9 \pm 5.6$ days, $p=0.01)$; decompensation of HF by poor adherence $(16.8 \%$ vs. $10.5 \% ; p=0.001)$; and in-hospital mortality ( $9 \%$ vs. $6.9 \% ; p=0.24)$

Conclusion: During the 2 years of the CCP there was a reduction of 1 day in the length of stay, and a lower frequency of hospitalizations by poor treatment adherence, and in readmissions in 30 days.

P9

Implication on the prognosis according to the cause of heart failure decompensation

Pedro Gabriel MB Silva*, Flavio Brito, Mariana Okada, Patricia Roveri,

Douglas Ribeiro, Giuliano Generoso, Jose Teixeira, Thiago Macedo,

Antonio Baruzzi, Valter Furlan

Hospital Totalcor, Cerqueira Cesar, São Paulo, São Paulo, Brazil

Critical Care 2015, 19(Suppl 2):P9; doi:10.1186/cc14665
Introduction: Heart failure (HF) is responsible for the majority of hospitalizations due to cardiovascular disease, and different clinical triggers are related to the cardiac decompensation.

Objective: To evaluate the prognosis of patients hospitalized due to acute HF, according to the cause of decompensation.

Methods: We retrospectively evaluated data from 731 patients consecutively admitted to a private cardiovascular center due to acute HF during 2013. We analyzed the frequency of each factor assigned as the trigger for the decompensation of HF among these patients, and also the length of stay and the number of deaths in each group. The infection group was compared with the other two groups separately, using Fisher's exact test for categorical variables and Student's $t$ test for continuous variables.

Results: The factor "infection" was associated with more days of hospitalization (Table 1), above the average of other triggers $(10 \times 6.95$ days; $p$ <0.01). The number of days in ICU in the cases of decompensation due to infection was also higher than the average from other causes $(5.8 \times$ 3.35 days; $p<0.01)$. In addition, of the 48 deaths in $2013,58 \%(n=28)$ were in patients with decompensated HF due to infection, and among these 28 deaths 15 were secondary to evolution of sepsis, in 6 there were predominance of the cardiac condition while the remaining 7 deaths showed mixed shock (cardiac and septic) or other complications related to both conditions leading to death. Conclusion: Infection was the main factor of decompensation, requiring a longer hospital stays, more days in the ICU and being responsible for most of the deaths occurred in patients hospitalized for acute HF. Studies of specific approaches in acute HF triggered by infection are warranted.

Conclusion: Infection was the main factor of decompensation, requiring a longer hospital stay, more days in the ICU and being responsible for most of the deaths occurring in patients hospitalized for acute HF. Studies of specific approaches in acute HF triggered by infection are warranted.

P10

Prognostic value of the hemolysis index in patients with significant hemolysis

Diego O Cortés*, Arthur Cezar M Xavier, Bruno R Almeida, Érica C Vieira, Jacques Creteur, Jean-Louis Vincent, Joao Claudio Lyra, Sylmara Zandona Department of Intensive Care, Hôpital Erasme, Anderlecht, Brussels, Belgium Critical Care 2015, 19(Suppl 2):P10; doi:10.1186/cc14666

Introduction: Hemolysis is a frequent complication of different extracorporeal circulation and membrane oxygenation (ECMO) support systems. Usually it is assessed by measuring the levels of haptoglobin or the concentrations of free hemoglobin in the plasma, but automated biochemical laboratory analyzers now detect the hemolysis index $(\mathrm{HI})$ of all blood samples as a measure of sample quality. We studied whether this simple index could detect populations at high risk of active hemolysis and whether it is correlated with outcome.

Methods: We evaluated all admissions to our department of intensive care during 2013 and collected relevant demographic and organ dysfunction data during the first 24 hours as required for the SOFA score (not the neurological component). We also collected data on whether or not the patients needed renal replacement therapy during the ICU stay. Patients

Table 1(abstract cc14664)

\begin{tabular}{|c|c|c|}
\hline & Pre-CCP (historical group) & CCP (intervention group) \\
\hline Number of patients & 338 & 1850 \\
\hline Mean age & $71( \pm 13.5)$ & $69( \pm 11.2)$ \\
\hline Male (\%) & $55 \%$ (95\% Cl: 50-60\%) & $56 \%$ (95\% Cl: 54-58\%) \\
\hline Mean EF (\%) & $37 \%( \pm 13.3)$ & $42 \%( \pm 11.1)$ \\
\hline Hemodynamic profile C (\%) & $5.65 \%$ (CI95\%: 3-8\%) & $4.2 \%(95 \% \mathrm{Cl}: 3.4-5.2 \%)$ \\
\hline Ischemic cardiopathy (\%) & 48.2\% (CI95\%: 43-53.5\%) & $58 \%$ (95\% Cl: 56-60\%) \\
\hline HFpEF (\%) & 37\% (CI95\%: 32-42\%) & $27 \%$ (95\% Cl: 25-29\%) \\
\hline Cardiorenal syndrome (\%) & 35\% (CI95\%: 30-40\%) & $33 \%$ (95\% Cl: 31-35\%) \\
\hline Infection (\%) & $23 \%$ (95\% Cl: 19-28\%) & $22 \%$ (95\% Cl: $20-24 \%)$ \\
\hline
\end{tabular}


Table 1(abstract cc14665)

\begin{tabular}{lllll}
\hline & Infection $(\boldsymbol{n}=\mathbf{2 5 3})$ & Noncompliance $(\boldsymbol{n}=\mathbf{1 2 6})$ & Progress of disease $(\boldsymbol{n}=\mathbf{1 9 1})$ & $\boldsymbol{p}$ value \\
\hline ICU stay (days) & $5.8( \pm 9)$ & $3.7( \pm 3.9)$ & $3.2( \pm 3.1)$ & 0.013 and $<0.001$ \\
Length of stay (days) & $10( \pm 9.2)$ & $7( \pm 5.7)$ & $8.1( \pm 7.3)$ & 0.019 and $<0.001$ \\
In-hospital mortality (\%) & 11.1 & 3 & 5.2 & 0.009 and 0.039 \\
Readmission in 30 days (\%) & 21.3 & $19.8 \%$ & 12 & 0.78 and 0.011 \\
\hline
\end{tabular}

Table 1(abstract cc14666) Characteristics and hemolysis index in different groups

\begin{tabular}{|c|c|c|c|c|}
\hline & ECMO $(n=56)$ & CBP $(n=246)$ & Others $(n=1719)$ & $p$ value \\
\hline Age (years) & $53(38-61)$ & $68(59-78)$ & $59(45-70)$ & $<0.01$ \\
\hline Emergency surgery, $n$ (\%) & $4(7)$ & $10(4)$ & $177(10)$ & $<0.01$ \\
\hline Lowest mean arterial pressure in first 24 hours $(\mathrm{mmHg})$ & $64(60-69)$ & $65(61-69)$ & $71(64-82)$ & $<0.01$ \\
\hline Norepinephrine use in first 24 hours, $n(\%)$ & $49(88)$ & $143(58)$ & $363(21)$ & $<0.01$ \\
\hline Renal replacement therapy during ICU stay, $n$ (\%) & $22(39)$ & $13(5)$ & $109(6)$ & $<0.01$ \\
\hline Transfusion during first 48 hours, $n(\%)$ & $41(73)$ & $76(31)$ & $223(13)$ & $<0.01$ \\
\hline Renal failure at admission, $n(\%)$ & $35(63)$ & $51(21)$ & $440(26)$ & $<0.01$ \\
\hline SOFA score at admission & $9(7-11)$ & $6(4-7)$ & $3(1-5)$ & $<0.01$ \\
\hline Mortality, $n(\%)$ & $26(46)$ & $15(6)$ & $186(11)$ & $<0.01$ \\
\hline Initial hemolysis index & $7(2-18)$ & $11(4-21)$ & $2(0-4)$ & $<0.01$ \\
\hline Median hemolysis index & $2(1-4)$ & $3(1-5)$ & $2(0-3)$ & $<0.01$ \\
\hline
\end{tabular}

were classified into three groups: those who needed ECMO support during the ICU stay, those who were admitted after cardiac surgery and had cardiopulmonary bypass (CPB), and other patients. We compared the initial and median (throughout the ICU stay) $\mathrm{HI}$ values in the different groups and the survivors with the nonsurvivors. We used SPSS 22.0 (IBM, USA) for all analyses and a $p$ value $<0.05$ was considered as significant.

Results: We studied 2021 patients with the characteristics presented in Table 1. Patients treated with ECMO and cardiac surgery patients had higher initial and median $\mathrm{HI}$ values than the other patients. The nonsurvivors in the ECMO group had higher median $\mathrm{HI}$ values than survivors (4 (2-21) vs. 2 (1-3), $\mathrm{p}<0.01)$. There were no differences in the initial or median $\mathrm{HI}$ values between patients treated or not with renal replacement therapy.

Conclusion: Patients undergoing CBP for cardiac surgery and those treated with ECMO support had higher HI values measured automatically in contemporary analyzers. The $\mathrm{HI}$ had prognostic value in patients treated with ECMO.

\section{P13}

Impact of multidisciplinary cardiology rounds on a cardiac ICU: a prospective cohort study

Rodrigo MV Melo*, Amilton S Junior, Fabio Gomes, Fernando Colombari, Fernando Zampieri

Intensive Care Unit, Hospital Alemao Oswaldo Cruz, Bela Vista, São Paulo, São Paulo, Brazil

Critical Care 2015, 19(Suppl 2):P13; doi:10.1186/cc14667

Introduction: Quality improvement is an important activity for all members of an interdisciplinary cardiology critical care team.

Objective: To evaluate whether multidisciplinary quality improvement in cardiac critical care, focused on a daily routine of rounds, protocol standardizations, and under the leadership of the same attending cardiologist in charge of coordinating a team, could produce better outcomes and resource utilization.

Methods: Prospectively collected data for consecutive patients who were admitted to a nine-bed cardiac intensive care unit (CICU) in two periods: January-June 2013 and January-June 2014. In the first period (control group) the patients were evaluated by common CICU routine, each day attended by a different intensivist physician, with no standardization of the multidisciplinary approach. Between the two periods there was a 6-month multidisciplinary training. In the second period (intervention group) the same cardiologist and multidisciplinary team made the daily routine rounds, with standardizations of managements and evidence-based care. Demographics and outcomes (mortality, time of ICU stay and mechanical ventilation time) were compared.

Results: A total of 610 patients were evaluated in the period of the study, $314(51.4 \%)$ in the control group and 296 (48.6\%) in the intervention group. Both groups were well matched for demographics: intervention and control group respectively, mean age of $68.9( \pm 14.7)$ vs. $70.9( \pm 13.2), p=$ 0.08 ; admission after cardiac surgery 21 (7.1\%) vs. $26(8.3 \%), p=0.40$ admission after percutaneous interventions 60 (20.3\%) vs. 59 (18.8\%), $p=$ 0.55 . The mean predicted mortality assessed by the simplified acute physiology score 3 (SAPS-3) and the Charlson comorbidity index were similar in both groups: intervention and control respectively, $43.1( \pm 13.1)$ vs. $42.8( \pm 12.9), p=0.66$ and $1.91( \pm 2.1)$ vs. $1.90( \pm 2.2), p=0.97$. Despite this, the mean ICU stay was lower in the intervention group as compared with the control group, $2.5( \pm 3.4)$ vs. $3.4( \pm 3.8)$ days, $p=0.003$; as was the mean mechanical ventilation time $0.84( \pm 0.16)$ vs. $4.16( \pm 1.47), p=0.005$ The 30-day mortality was 11 (3.7\%) vs. 15 (4.7\%), RR 0.79, 95\% Cl 0.64-3.03, $p=0.40$. After multivariate analysis, there were no changes in the results. Conclusion: A focused cardiac critical care management on a CICU, based on a multidisciplinary approach and daily rounds performed by the same cardiologist, reduced the CICU stay and mechanical ventilation time, with the same mortality rates. This action could help improve resource utilization.

P14

Theoretical-practical training of students from high school to care for cardiac arrest: a prospective study

Vanessa F Marçolla*, Edna R Santos, Ivana PB Aragão, Karina R Silva, Kleison P Silva, Lorhana VB Oliveira, Marcio Canedo, Raphaela B Gomes

Rio de Janeiro State Government, Santa Cruz, Rio de Janeiro, Rio de Janeiro, Brazil Critical Care 2015, 19(Suppl 2):P14; doi:10.1186/cc14668

Introduction: Cardiovascular diseases are the leading cause of death in the world, and sudden cardiac arrest is a major contributor to this index. Training reduces ignorance and fear, increasing safety to recognize that the victim is not breathing properly, so as to trigger help and start CPR as soon as possible.

Objective: To apply theoretical-practical training to a vocational public high school, so as to work correctly, quickly and safely before cardiopulmonary arrest, resuscitation maneuvers running efficiently, in order to save lives. 
Methods: This study was designed as a prospective investigation of 1800 students from a vocational public high school, between 2012 and 2013. The program of theoretical and practical training lasts 2 hours. Each student attends a lecture with a video on the subject for 30 minutes after 30 minutes of classroom practice. Then, using a practical training mannequin, they are assessed through a performance checklist. A questionnaire was distributed before the start of training to see whether the student had prior knowledge about a rescue in the event of cardiac arrest.

Results: More than $50 \%$ did not have any knowledge about the subject. This evaluation showed that after 2 hours of training and analyzing the performance checklists: $85 \%$ knew how to perform the procedures and call for help effectively, $30 \%$ were able to recognize the absence of breathing and $35 \%$ positioned themselves and began chest compressions as recommended form.

Conclusion: Students from the school showed that $90 \%$ of adolescents when trained are able to act at the scene of a cardiac arrest, multiplying the knowledge to family and community to save lives. However, according to the international recommendations, retraining as an ideal does not exceed 2 years.

P15

Admission factors associated with ICU readmission in oncohematological critically ill patients: a retrospective cohort study Leandro U Taniguchi", Cinthia M Rodrigues, Ellen MC Pires, Jorge PO Feliciano, Jose M Vieira Jr

Education and Research Institute, Hospital Sírio-Libanês, São Paulo, São Paulo, Brazil Critical Care 2015, 19(Suppl 2):P15; doi:10.1186/cc14669

Introduction: Despite initial recovery, many onco-hematological patients require ICU readmission, which is a burdensome condition with implications both for mortality and quality of life. Nevertheless, little has been published regarding identification of risk factors for ICU readmission in the oncohematological population of critically ill patients.

Objective: To determine the admission factors associated with ICU readmission among onco-hematological patients.

Methods: Retrospective cohort study using an ICU database (Sistema Epimed $^{T M}$ ) from a tertiary oncological center. From January 2012 to December 2012, 2629 patients were admitted to our ICU. Forty-nine percent (1155 patients) had onco-hematological conditions and were the subject of this retrospective study. We used univariate and multivariate analysis to identify at admission risk factors associated with later ICU readmission in the same hospitalization period.

Results: One hundred and five patients $(9.1 \%)$ were readmitted after ICU discharge. Patients readmitted were sicker compared with the nonreadmitted group (SAPS III of 49 (IQR 33-53) vs. 37 (28-49) respectively, $p<0.001$ ), had a nonsurgical reason for hospitalization (79\% vs. $40 \%$ respectively, $p<0.001)$, more frequently came from the ward $(36.2 \%$ vs. $13.9 \%$ respectively, $p<0.001$ ), had longer hospital length of stay (LOS) before ICU admission (15 days (8.5-29) vs. 1 day $(0-2)$ respectively, $p<0.001)$, more frequently came to the ICU due to respiratory failure $(19.2 \%$ vs. $4.7 \%$ respectively, $p<0.001)$ or neurological disturbances $(24 \%$ vs. $8 \%$ respectively, $p<0.001)$, more frequently required mechanical ventilation $(34.6 \%$ vs. $18.4 \%$ respectively, $p<0.001)$ and vasoactive drugs $(47.1 \%$ vs. $33.3 \%$ respectively, $p<0.001)$, and had higher hospital mortality $(28.6 \%$ vs. $13.9 \%$ respectively, $p<0.001)$. On multivariate analysis, longer hospital LOS before ICU transfer, neurological disturbances and nonsurgical reason for admission were independent risk factors for ICU readmission.

Conclusion: We identified some risk factors associated with ICU readmission in onco-hematological patients, but unfortunately all are not amenable to interventions. Identification of risk factors at ICU discharge might be more promising.

\section{P16}

Appraisal of the daily clinical rounds performed in an open and general ICU Roberto R Filho*, Ana Maria Cavalheiro, Denise C Cazati, Frederico Lomar, Gustavo FJ de Matos, Murillo SC de Assunção, Roberto R Filho,

Tatiana Mohovic, Thiago D Correa

Department of Intensive Care Medicine, Hospital Israelita Albert Einstein,

Morumbi, São Paulo, São Paulo, Brazil

Critical Care 2015, 19(Suppl 2):P16; doi:10.1186/cc14670
Table 1(abstract cc14670) Main interventions proposed and performed during the multiprofessional rounds

\begin{tabular}{lll}
\hline Main intervention & Proposed & Performed \\
\hline Changes in nutritional support & $39 / 158(24.7 \%)$ & $39 / 39(100.0 \%)$ \\
Early mobilization & $37 / 158(23.4 \%)$ & $35 / 37(94.6 \%)$ \\
Adjustments on sedation or analgesia & $51 / 158(32.3 \%)$ & $49 / 51(96.1 \%)$ \\
Adjustments on & $26 / 158(15.8 \%)$ & $26 / 26(100.0 \%)$ \\
Withdrawal of invasive devices & $23 / 158(14.6 \%)$ & $19 / 23(82.6 \%)$ \\
Adjustments of ventilatory parameters & $23 / 158(14.6 \%)$ & $23 / 23(100.0 \%)$ \\
Adjustments on glycemic control & $25 / 158(14.6 \%)$ & $25 / 25(100.0 \%)$ \\
Deep vein thrombosis prophylaxis & $13 / 158(8.2 \%)$ & $12 / 13(92.3 \%)$ \\
Stress ulcer prophylaxis & $6 / 158(3.8 \%)$ & $5 / 6(83.3 \%)$ \\
\hline
\end{tabular}

Values represent $n$ (\%)

Introduction: Daily multidisciplinary clinical rounds involving physicians, nurses, respiratory therapists, nutritionists and clinical pharmacists improve the quality and outcomes of ICUs [1]. However, data regarding performance of these clinical rounds in an open-ICU model are limited. Objective: To address the characteristics and the main interventions proposed and made during multiprofessional clinical rounds performed in a clinical-surgical open ICU.

Methods: This observational study was conducted in a 41-bed open clinical-surgical ICU of a tertiary-care, private hospital in São Paulo, Brazil. From February 20 through March 28 2013, demographic data, SAPS 3, the participants of the ICU clinical rounds, the number and type of the proposed interventions, and the number of performed interventions by the multidisciplinary team were recorded and analyzed.

Results: A total of 158 clinical rounds were included in this analysis. Fiftyfour percent (85/158) of the patients were male with median (IQR) age of $73(60-84)$ years and SAPS 3 score of $52(44-65)$. The multidisciplinary team was composed of a senior physician $(157 / 158(99 \%))$, nurses $(157 / 158$ $(99 \%))$, an on-call staff physician $(150 / 158(95 \%))$, respiratory therapists $(149 / 158(94 \%))$, a clinical pharmacist $(89 / 158(56 \%))$ and nutritionists $(62 / 158(39.2 \%))$. The median (IQR) number of interventions proposed during the multidisciplinary rounds was $1(0-2)$ and the number of performed interventions was 1 (0-2) (Table 1). Interventions were more frequently proposed by senior physicians $(82 / 158(52 \%))$ followed by respiratory physiotherapists (43/158 (27\%)) and a clinical pharmacist (29/158 (18\%)).

Conclusion: In our open ICU model where decisions should be shared with assistant doctors, the implementation of daily clinical rounds was associated with an intense participation of the multidisciplinary team and with a high level of performance of the proposed interventions. These actions are probably associated with better care of the critically ill patients. However, further studies are needed to correlate such interventions with clinical outcomes.

References

1. Kim MM, Barnato AE, Angus DC, Fleisher LA, Kahn JM: The effect of multidisciplinary care teams on intensive care unit mortality. Arch Intern Med 2010, 170(4):369-376.

2. Weiss $C H$, Moazed F, McEvoy CA, Singer BD, Szleifer I, Amaral LA, et a: Prompting physicians to address a daily checklist and process of care and clinical outcomes: a single-site study. Am J Respir Crit Care Med 2011, 184(6):680-686.

P17

Analysis of ICU physician predictions for ICU length of stay Antonio Paulo N Junior*, Pedro Caruso

A.C. Camargo Cancer Center, Liberdade, São Paulo, São Paulo, Brazil Critical Care 2015, 19(Suppl 2):P17; doi:10.1186/cc14671

Introduction: An adequate prediction of ICU length of stay (ICU LOS) is of paramount importance to adequately allocate resources and inform patients and families. However, literature evaluating ICU physician predictions on ICU LOS are sparse. 
Methods: Patients admitted to four medical-surgical ICUs in an oncology teaching hospital in 2014 were included. As part of the admission form, the physician responsible for patient admission is asked to inform an estimate of ICU LOS for that patient (less than 48 hours, 2-5 days or more than 5 days). Agreement of predicted and actual ICU LOS was calculated. Patients and physician characteristics at admission were evaluated to identify associated factors for underestimation and overestimation of ICU LOS. Two logistic regression analyses were performed to identify independent risk factors for each outcome.

Results: A total of 2955 patients were admitted during the study period (female sex: $46.5 \%$, median age: 61 (51-71) years). Admissions were mainly for elective surgery (56.5\%), followed by medical reasons (37\%). Readmissions encompassed $9.3 \%$ of total admissions. Median SAPS 3 was 48 (40-64) and ICU mortality was 8.5\%. Median ICU LOS was 2 (1-3) days. Physicians adequately predicted ICU LOS in $53 \%$ of admissions. ICU LOS were underestimated in $29 \%$ and overestimated in $18 \%$ of cases. Kappa statistics was 0.222 (0.195-0.249). Sex, scheduled surgical admission, Eastern Cooperative Oncology Group (ECOG) performance status, mechanical ventilation, vasopressor use and active infection at admission were associated with underestimation of ICU LOS. Male sex (OR $=0.80$; $95 \% \mathrm{Cl} 0.65-0.98)$, and active infection at admission (OR $=1.35 ; 95 \% \mathrm{Cl}$ 1.02-1.78) were independently associated with underestimation of ICU LOS in logistic regression. Type of admission (medical and urgent surgery), reason for admission (not postoperative monitoring), ECOG, mechanical ventilation, vasopressor use, delirium and infectious status at admission, SAPS 3, serum creatinine and being readmitted were associated with overestimation of ICU LOS. Type of admission (OR $=0.64$; $95 \% \mathrm{Cl} 0.51-0.80)$, reason for admission (OR $=0.93 ; 95 \% \mathrm{Cl} 0.87-0.99)$, ECOG (OR $=0.84 ; 95 \% \mathrm{Cl} 0.76-0.92)$ and active infection at admission (OR $=0.60$ ) were independently associated with overestimation of ICU LOS in logistic regression.

Conclusion: ICU physicians adequately predicted ICU LOS in only 53\% of admissions. Sex and active infection were independently associated with underestimation of ICU LOS. Type of and reason for admission, ECOG, ventilator support and active infection at admission were independently associated with overestimation of ICU LOS.

P18

Basic life support training for lay students from a public university

Silmara Meneguin*, Debora G de Santana, Larissa M Noda

Medical School of Botucatu, UNESP, Distrito de Rubião Jr S/N, Botucatu, São

Paulo, Brazil

Critical Care 2015, 19(Suppl 2):P18; doi:10.1186/cc14672

Introduction: Emergency care situations come up in people's lives unexpectedly, demanding rapid, objective and effective action. People are not always properly trained to deliver this care though.

Objective: To attend to this shortage, this study compared the psychomotor skills and theoretical knowledge of lay students in the cardiopulmonary resuscitation (CPR) technique before and after training.

Methods: Concurrent multiple cohort study, based on a sample of 424 students from a Brazilian public university who participated in on-campus training between July 2012 and December 2014. To assess the theoretical knowledge, a structured questionnaire was used and, to assess the psychomotor skills, a checklist with the steps of the CPR technique, in line with the 2010 guidelines of the American Heart Association. The participants were divided into four knowledge areas: biological, exact, agricultural and health.

Results: These study results evidenced a statistically significant increase in correct answers on the theoretical knowledge and psychomotor skill assessment tools after the training in relation to the areas. Exact and agricultural sciences were the areas that most evolved in terms of the number of correct psychomotor skills. Agricultural and biological sciences were the areas that most evolved regarding theoretical knowledge about cardiopulmonary resuscitation. Before the training, the mean number of correct skills was an average 1.79 points higher for each additional year of age and the men's score was 6.6 points lower than the women's score. After the course, only the relation between age and number of correct skills continued significant and gained strength. For each additional year of age, the number of correct skills increased by an average 8.21 points. As regards the theoretical knowledge score on CPR, before the course, a significant relation existed between age and having taken the first aid course. The score increased by 0.22 points for each additional year of age and was 0.63 points higher among participants who had taken the course earlier. After the training, sex and having taken the course earlier remained significantly related with the theoretical knowledge score on CPR.

Conclusion: These study results indicate that the participants have presented improvements in their performances. After the training, the increase in the number of correct answers on the psychomotor skill tool was directly proportional to the age. Concerning the theoretical knowledge on CPR after the course, age and having taken the first aid course contributed to increasing the number of correct answers.

P19

Caring for critically ill patients outside ICUs due to a full unit

Abimael C Silva*, Camila B Oyama, Cintia MC Grion, Eduardo H Rodrigues, Fabiane Urizzi, Lucienne TQ Cardoso, Renata G Oliveira, Thalita B Talizin Universidade Estadual de Londrina, Vila Operária, Londrina, Paraná, Brazil Critical Care 2015, 19(Suppl 2):P19; doi:10.1186/cc14673

Introduction: Advances in medicine allow patients with comorbidities to live longer and the need for intensive care increases. It is not unusual for the supply of critical care beds to not meet the demand. In this scenario the critically ill patient is cared for in the emergency department or in regular hospital wards.

Objective: To describe clinical and epidemiologic characteristics of critically ill patients treated outside the ICU due to an unavailability of beds.

Methods: Prospective cohort study of critically ill patients treated in the hospital wards of a university hospital during a 1-year period. Al consecutive patients denied ICU beds due to a full unit who were treated by hospital ward staff and daily intensivist physician consultation during the period February 2012-February 2013 were included. Patients were followed until admission to the ICU or cancellation of the ICU bed request. Clinical and epidemiologic data were collected. Invasive procedures and therapeutic interventions were noted. Outcome was observed at hospital discharge.

Results: Four hundred and fifty-four patients were analyzed. Patients were predominantly male $(54.6 \%)$ and the median age was 62 (interquartile range (ITQ): 47-73). Median APACHE II score was 22.5 (ITQ: 16-29), median SOFA score was 8 (ITQ: 4-13) and median TISS 28 was 27 (ITQ: 22-30). Reasons for critical care request were respiratory failure (39\%), hemodynamic instability (36.3\%), neurologic monitoring (14.5\%), cardiac monitoring (7.3\%) and postoperative care $(2.9 \%)$. Invasive mechanical ventilation was used in 266 (65.6\%) patients, continuous intravenous vasopressors or inotropic drugs for shock treatment were used in $44.9 \%$ and intravenous vasodilators in $5.9 \%$ of patients. Median time of follow up was 3 (ITQ: 2-6) days, after this time 204 patients were admitted to the ICU and 250 had the ICU bed request cancelled. The motives for waiving critical care were due to clinical improvement in $122(26.9 \%)$ patients, death in $101(22.3 \%)$ patients, decision to withhold treatment due to futility in $25(5.5 \%)$ patients and transfer to another institution in two (0.4\%) patients. Hospital mortality was $65 \%$.

Conclusion: Caring for critically ill patients outside ICU walls was frequent in the study institution. Patients presented a high severity of disease score, had multiple organ dysfunctions and needed multiple invasive therapeutic interventions. Despite receiving intensive care with specialized consultation, these patients present poor prognosis.

P20

Chronic critical illness: reason for concern during and after ICU admission!

Carlos Augusto R Feijó *, Allison EPP Borges, Eduardo Q da Cunha,

Francisco A de Meneses, Marina P Albuquerque, Natália LP Aragão,

Tamara O Pinheiro, Túlio S de Aguiar

General Hospital of Fortaleza, Papicu, Fortaleza, Ceará, Brazi

Critical Care 2015, 19(Suppl 2):P20; doi:10.1186/cc14674

Introduction: Evolution of healthcare assistance in the ICU, due to its technological apparatus, has increased survival of critically ill patients 
substantially. In this ballast, patients who require prolonged length of stay, as well as demand continued intensive care support (particularly mechanical ventilation (MV)), are increasingly identified. These patients suffer from chronic critical illness (CCI), a rapidly growing syndrome which has immune, neuroendocrine and metabolic particularities-today, they represent $5-15 \%$ of all patients admitted to the ICU.

Objective: To describe the epidemiological characteristics of $\mathrm{CCl}$ in an adult ICU and to analyze possible predicting factors of evolution to $\mathrm{CCl}$ at admission to the ICU.

Methods: Retrospective analysis of patients admitted to the General Hospital of Fortaleza-SESA from November 2014 to February 2015. The CC was defined as prolonged MV (>21 days, for at least 6 hours per day). Descriptive statistical analysis was utilized for demographic characters, $t$ test for evaluation of continuous variables and chi-square test for categorical variables.

Results: From 86 patients admitted in that period, 13 (15\%) were identified as chronically critical. The mean age of these patients was $57 \pm$ 22 years, and $61 \%$ of them were female, mean SOFA score (at admission) was $6.5 \pm 3.3$ points and mean APACHE II score was $18 \pm 5.5$ points, statistically similar to the other patients. At admission, $61.3 \%$ had hypertension, $46.1 \%$ were diabetics; chronic kidney disease (CKD) and chronic cardiovascular disease were identified in $23 \%$ each. The presence of diabetes mellitus (DM) and CKD were significantly higher in the CCl population ( $p=0.048$ and $p=0.033$, respectively). The mean MV time, ICU and hospital length of stay were significantly increased in the $\mathrm{CCl}$ population, with $34 \pm 12$ days $(p<0.001)$.

Conclusion: The prevalence of $\mathrm{CCl}$ in this study follows the current literature. High SOFA and APACHE II scores do not seem able to identify the patients who will evolve from severely ill to $\mathrm{CCl}$. The present study suggests that comorbidities, at admission, may indicate which patients will survive acute critical illness to become CCl-as well as CKD and DM. The significant post-ICU mortality highlights the need for more suitable followup for the $\mathrm{CCl}$ population after discharge.

P22

\section{Patients treated outside ICUs}

Ana Vitoria CS Gasparine*, Cintia MC Grion, Claudia MDM Carrilho,

Fabiane Urizzi, Joao Paulo M Favoreto, Patricia R Pêras, Patricia S Moya, Raquel Mireski

Universidade Estadual de Londrina, Vila Operária, Paraná, Brazil

Critical Care 2015, 19(Suppl 2):P22; doi:10.1186/cc14675

Introduction: Intensive care beds are scarce resources, particularly in developing countries. For this reason it is not uncommon for critically ill patients to be treated in emergency rooms or in hospital wards due to unavailability of intensive care beds.

Objective: To describe direct costs of treatment of critically ill patients treated outside ICUs due to a full unit.

Methods: Prospective cohort study of critically ill patients treated outside the ICU by the ward health care staff with daily intensivist physician consultation in a university hospital during a 6 -month period. All consecutive patients who were treated outside the ICU due to a full unit during the period of February 2012-February 2013 were included in the study. Patients were followed daily until they were transferred to an ICU bed or the request for intensive care was cancelled. Clinical and demographic data were collected to characterize the study sample and data to calculate costs using the bottom-up method were collected and grouped into four categories: clinical support, consumable items, human resources and hospital taxes. The Brazilian Medical Association (AMB) price index for medical procedures and the BRASINDICE price index for medications, solutions and hospital consumables were used to calculated costs.

Results: One hundred and fifty-one patients were included in the study. Median age was 64 (interquartile range (ITQ): $49-72$ ) years and 55\% were male. Median APACHE II score was 23 (ITQ: 16-29), median SOFA score was 8 (ITQ: 4-11) and median TISS 28 was 27 (ITQ: 21-30). The median time that patients were observed was 3 (ITQ: 2-6) days, after this period of time patients were either admitted to the ICU or the request for an ICU bed was cancelled. Median total cost per patient treatment was $R$ $\$ 12,846.29$ (ITQ: R\$8279.00-21,763.61), and median daily cost was $R$ \$3496.80 (ITQ: R\$2668.84-4391.01). Total costs for nonsurvivor patients were higher $(\mathrm{R} \$ 13,307.08)$ compared with survivors ( $\mathrm{R} \$ 10,835.53, p=$ 0.041). Hospital mortality was $66.1 \%$.

Conclusion: Treatment of critically ill patients outside the ICU is costly and associated with poor prognosis. Direct costs of treatment of critically ill patients outside the ICU are higher in nonsurvivors.

P23

Does the admission time make a difference in outcome?

André Negrão*, Bruno F Mazza, Débora D da S Mazza,

Sebastião César de Vasconcellos

Hospital São Luiz, Rede D’Or, Unidade Morumbi, São Paulo, São Paulo, Brazil Critical Care 2015, 19(Suppl 2):P23; doi:10.1186/cc14676

Introduction: The number of hospital admissions during the night is a problem in ICUs. The high occupancy rate and the high demand for beds causes the inflow of patients to occur at night in most cases. The adequacy of the health care team must be made with the intention of providing the best patient care. For this, it is important to have knowledge of hospital dynamics in the unit, so you can optimize the team for this purpose.

Objective: The objective of this study was to evaluate whether there are differences in the outcome of patients admitted during the day compared with those admitted during the night.

Methods: A retrospective analysis was performed from January to December 2014, using the database EPIMED ${ }^{\bullet}$. We evaluated 3186 patients who were admitted to hospital ICUs. We evaluated the epidemiological characteristics of patients and the outcome of them. We classified the patients into two groups of patients: group 1 was admitted to the ICU during the day (7:00 a.m.-7:00 p.m.) and group 2 at night (7:00 p.m.-7:00 a. m.). Statistical analysis was performed using SPSS 22 software. The Student $t$ test was used to analyze numerical variables and the chi-square test to analyze the categorical variables. We considered data statistically significant if $p<0.05$.

Results: There was a higher number of admissions to the ICU at night $2154(67.6 \%)$ vs. $1032(32.4 \%)$. The principal source of patients was the emergency room, 2372 (74.5\%) patients. There are more clinical than surgical patients, $82.2 \%$ vs. $17.8 \%$. There were no significant differences between groups regarding demographic variables and severity scores. The SAPS 3 score was $41.67 \pm 11.44$ in group 1 and $41.81 \pm 12.51$ in group $2(p=0.75)$. There was no difference in length of stay in the ICU, group 1: $4.61 \pm 7.54$ vs. group 2: $4.83 \pm 7.23(p=0.42)$. There was no difference in ICU, group 1: 91 (4.2\%) vs. group 2: 55 (5.6\%), or hospital, group 1: 164 (8.2\%) vs. group 2: 98 (10.4\%), mortality between groups, $p=\mathrm{NS}$.

Conclusion: In our study, we found that a high admission rate occurs at night. The ICU team needs to be adjusted for this demand. There is no difference between groups in severity, length of stay and ICU or hospital mortality in this group of patients.

P24

Evaluation of residual urine volume after catheterization by ultrasound performed by a nurse

Ana Maria Cavalheiro*, Debora S da S Alves, Dejanira Aparecida Regagnin,

Fabio Augusto C Vieira, Leonardo L Rocha, Maria Teresa Ap S Odierna,

Maura Cristina dos Santos, Neide M Lucino

Hospital Albert Einstein, Morumbi, São Paulo, São Paulo, Brazil

Critical Care 2015, 19(Suppl 2):P24; doi:10.1186/cc14677

Introduction: The risk factors described associated with urinary tract infection (UTI) during urethral catheterization are the colonization of the urinary tissue during evaluation. The incidence of UTI is directly related to the duration of the catheterization, and, after considering this everpresent factor in multivariate analysis, it is the main and most important risk factor for UTI. Thus, a simple noninvasive method for determining the volume of urine in the bladder would be very well received. As such, it is possible for properly trained nursing staff to use ultrasound as a vehicle for the evaluation of residual urinary volume, and Brazilian institutions have the potential to gain much based on surveys already conducted. Objective: To analyze the duration of time Foley catheters are used and then replace with use of ultrasound scanning to evaluate residual urine volume. 
Methods: This is a quantitative study, assessed by the ethics committee of Albert Einstein Israelita Hospital, protocol number 2267601380000071. In the first phase, 20 nurses were allowed to evaluate the residual urine volume with ultrasound and create proper care guidelines. After being taught these guidelines, nurses were allowed to remove Foley catheters from patients showing no signs of diuresis for at least 4 hours. The resulting identification of urinary volume was communicated to the attending physician and subsequent actions were determined by him. The information was recorded in a manner established in advance with volume data, volume drained, medical procedure, gender, age, reason for admission, and duration of time with indwelling bladder catheter all evaluated.

Results: An ultrasound evaluation of residual urine volume after catheterization was performed on 94 patients in the ICU. Thirty-one of these were surgical patients and 63 were clinical patients. Thirty-six were female and 58 were male. The Foley catheter was used for an average period of 3 days (DP 1.2). The average time for the nursing team to evaluate residual urine volume was 6 hours (DP 2.3). Fifty-eight out of the 94 patients studied exhibited spontaneous diuresis after mechanical stimulation such as a change in position on the bed, use of cold or warm bags, and stimuli from the ultrasound transducer during examination and abdominal massage. Foley catheters were used in 12 patients who had more than $1000 \mathrm{ml}$ urine retention 4 hours after removal of the catheter. It was recommended to use the Nelaton catheter in 28 patients with an average urinary volume of $300 \mathrm{ml}$. Of the 94 patients studied, none exhibited signs of UTI during their hospital stay.

Conclusion: The results of this study show that the use of ultrasound as a tool for nurses to reduce Foley catheters in critically ill patients is an effective strategy to avoid ICU. Ultrasound is an effective nursing tool that safely and efficiently assesses urine volume within the bladder without the need for catheter insertion.

\section{P25}

Evaluation of the adverse events on applying a functional protocol in patients in an ICU

Maíra J Maturana*, Ana Paula O Rodrigues, Gabriela DA Martinelli, Layla H Amarantes, Luiz Alberto M Knaut, Paula TG Serra, Esperidião E Aquim 1-Faculdade Inspirar, Curitiba-Pr, São Francisco, Curitiba, Paraná, Brazil Critical Care 2015, 19(Suppl 2):P25; doi:10.1186/cc14678

Introduction: ICU patients are exposed to adverse events, which are defined as unintended complications but are preventable.

Objective: To identify the adverse effects on the application of the Prófisio Functional Physical Therapy Protocol in critical patients.

Methods: Experimental study, longitudinal and contemporary, taking place between January and October 2014 with patients admitted to the ICUs of the Trabalhador, Vita Curitiba, Vita Batel, Marcelino Champagnat Hospital and the Neurology Institute of Cutitiba (INC) in the city of Curitiba, PR. The sample was composed of 375 patients, being $57 \%$ male and $42.6 \%$ female, with an age average of $58 \pm 20.9$, medium Glasgow and Ramsey 5. The Prófisio Functional Physical Therapy Protocol (Table 1) was applied once a day to patients who were age 18 or older, hemodynamically stable with PAM between 60 and $110 \mathrm{mmHg}$, whose responsible agreed to sign the TCLE. The hemodynamic variables (heart rate, blood pressure, breathing rate and oxygen saturation) were evaluated before and after the application of the protocol. The adverse effects were defined as loss of central or peripheral venous access, electrodes for cardiac monitoring, intracranial pressure monitoring, external ventricular derivation, removal of urinary catheter, removal of gavage, orthotracheal or tracheostomy tubes, surgical drains, bleedings, and decrease and opening of sutures, and were observed during all application of the protocol.

Results: A total of 1144 interventions were observed, where only seven $(0.61 \%)$ showed adverse events. Of the seven only adverse effects, three were classified as light-loss of electrodes of cardiac monitoring-and four were classified as moderate-the unscheduled removal of the gavage, hypotension, drop and loss of surgical drain. The hemodynamic variables did not suffer significant alterations.

Conclusion: The application of the Prófisio Functional Physical Therapy Protocol showed itself to be safe and with a low risk of adverse effects, when applied to critical patients.

References

1. Souza GF, Albergaria TF, Bomfim N, Duarte AM, Fraga HM, Prata Martinez B: Eventos adversos do ortostatismo passivo em pacientes críticos numa unidade de terapia intensiva. Assobrafir Ciência, São Paulo 2014, 5(2):25-33

2. Korupolu R, Gifford J, Needham DM: Early mobilization of critically ill patients: reducing neuromuscular complications after intensive care. Contemporary Critical Care 2009, 6(9):1-11.

P26

Evaluation of the characteristics of older patients admitted to a general ICU

Bruno F Mazza*, Ana Lúcia V Ronchini, Dario F Ferreira, Joely L Malachia, Rosa GA Rocha, Samantha L Almeida

Hospital Samaritano, Higienópolis, São Paulo, São Paulo, Brazi Critical Care 2015, 19(Suppl 2):P26; doi:10.1186/cc14679

Introduction: Aging is a fact that occurs today, and is associated with increased prevalence of chronic diseases and functional impairment. This leads to an increase in hospitalization, especially in ICUs. A better understanding of the characteristics of these patients is essential to provide the best assistance we can and to have the best of the resources needed for the proper treatment of these patients [1].

Objective: The aim of this study is to compare the epidemiological characteristics of older patients ( $>70$ years) with those with lower age ( $<70$ years), admitted to a general ICU.

Methods: A retrospective analysis was performed from June to December 2014, using the database EPIMED ${ }^{\star}$. We evaluated 758 patients who were hospitalized in two ICUs, corresponding to 37 beds. Statistical analysis was performed using SPSS 22, using the Student $t$ test for numerical variables

Table 1(abstract cc14678) The Prófisio Functional Physical Therapy Protocol

\begin{tabular}{|c|c|c|c|c|c|}
\hline & Level 1 & Level 2 & Level 3 & Level 4 & Level 5 \\
\hline $\begin{array}{l}\text { A. COGNITIVE } \\
\text { CHANGES } \\
\text { Glasgow }<13\end{array}$ & $\begin{array}{l}\text { Electrostimulation; } \\
\text { Passive } \\
\text { Kinesiotherapy }\end{array}$ & $\begin{array}{l}\text { Postural control; } \\
\text { Passive bicycle; } \\
\text { Orthostatic board; } \\
\text { Outside the ICU ride }\end{array}$ & $\begin{array}{l}\text { Assisted } \\
\text { orthostatism }\end{array}$ & $\begin{array}{l}\text { not } \\
\text { applicable }\end{array}$ & not applicable \\
\hline $\begin{array}{l}\text { B. COGNITIVE } \\
\text { PRESERVED } \\
\text { Glasgow } \geq 13\end{array}$ & $\begin{array}{l}\text { Prevention of deep vein } \\
\text { thrombosis (PTVP); } \\
\text { Active-assisted kinesiotherapy; } \\
\text { Game therapy }\end{array}$ & $\begin{array}{l}\text { Postural control; } \\
\text { Active bicycle; } \\
\text { Orthostatic board; } \\
\text { Outside the ICU ride }\end{array}$ & $\begin{array}{l}\text { Standing position, } \\
\text { Kinesiotherapy } \\
\text { Weathered; } \\
\text { Transfer of weight } \\
\text { training; } \\
\text { March } \\
\text { Stationary }\end{array}$ & $\begin{array}{l}\text { Training } \\
\text { march } \\
\text { with } \\
\text { aid; } \\
\text { Squat }\end{array}$ & $\begin{array}{l}\text { Step; } \\
\text { Trampoline; } \\
\text { March } \\
\text { without } \\
\text { assistance; } \\
\text { One-leg } \\
\text { support }\end{array}$ \\
\hline Note & check specific contraindications & $\begin{array}{l}\text { At each level adding exercise described } \\
\text { in the table }\end{array}$ & & & \\
\hline
\end{tabular}

Level 1 bedridden/restricted to bed due to medical orders, Level 2 transfer bed-chair passively, Level 3 transfer-bed chair with partial weight, Level 4 rambles with partial weight, Level 5 wandering without assistance 
and the chi-square test for categorical variables. We considered statistically significant a $p$ value $<$ p. 05 .

Results: Patients older than 70 years accounted for $50 \%$ of admissions; $68 \%$ of clinical admissions and $32 \%$ surgical hospitalizations-when compared for the group $<70$ years, we have $60 \%$ of clinical admissions and $40 \%$ surgical ( $p=0.15$ ), with $50 \%$ of these coming from the emergency room. There was no difference between groups in length of stay in the ICU (>70: $6.73 \pm 7.76$ vs. $70: 12.0 \pm 22.3$ vs. $<70: 244.3 \pm$ 18.38) ( $p=$ NS). The group $>70$ years, however, showed a higher value of SAPS $3(55.6 \pm 15.12$ vs. $41.6 \pm 16.2, p=0.01)$ and a greater likelihood of death (39\% vs. $19.5 \%)$. Group > 70 years was higher $(13 \%$ vs. $8 \%, p=0.01$ ); however, the standardized mortality rate (SMR) for the group $>70$ years was 0.49 and for the group $<70$ was 0.45 .

Conclusion: In our study, we found a high hospitalization rate in patients older than 70 years in the unit, despite a higher SAPS 3, and no difference in length of stay in the ICU or hospital was shown. The group $>70$ years had a higher mortality but the adjusted mortality rate shows a good performance in both groups of patients. Increasing age is a fact and it is important be prepared to manage this kind of patient to give them the best possible care.

Reference

1. Bagshaw SM, Webb SA, Delaney A, George C, Pilcher D, Hart GK, et al: Very old patients admitted to intensive care in Australia and New Zealand: a multi-center cohort analysis. Crit Care 2009, 13(2):R45.

P27

Functional independence profile of critically ill patients

Karina Tavares Timenetsky*, José AS Junior, Andréia SA Cancio,

Angela SY Yang, Carolina SA Azevedo, Cilene SM Silva, Corinne Taniguchi, Daniela Nobrega, Fernanda Domingues, Juliana Raimondo,

Louise HR Gonçalves, Pedro Veríssimo, Raquel AC Eid

Hospital Israelita Albert Einstein, Morumbi, São Paulo, São Paulo, Brazil

Critical Care 2015, 19(Suppl 2):P27; doi:10.1186/cc14680

Introduction: The functional independence measure (FIM) is an outcome measure of the severity of physical and cognitive disability for an inpatient rehabilitation setting. The severity of disability changes during rehabilitation treatment, making changes in the FIM scale an indicator of treatment benefits and its results. So far there is no evidence for the functional profile of patients followed by physiotherapists during their critically ill department stay.

Objective: This study objective was to evaluate the functional profile of critically ill patients during their hospital stay through the FIM scale.

Methods: A cohort study evaluating the FIM scale of critically ill patients admitted to Hospital Israelita Albert Einstein, older than 18 years old, during a 1-year period (January-December 2014), that were followed by physiotherapists. The FIM scale was applied by trained and certified physiotherapists at day 1 (when the patient was alert and responsive), at ICU discharge and at critically ill department discharge. The FIM scale rates from 18 to 126 points, evaluating: self-care, sphincter control, transfer and mobility, deambulation, communication and social cognition. With the FIM scale, patients were characterized as independent, modified dependence, or complete dependence.

Results: A total of 1457 patients were included in the study, mean age of $85( \pm 4.5)$ years. Of these patients, $56 \%$ were male. The most frequent admission diagnosis was respiratory disorders in $28.4 \%$ of the patients, followed by $18 \%$ cardiac reasons, $16.4 \%$ infection, and $11.5 \%$ neurological disorders. The median FIM scale was 62 points (48-81) at day 1 and 83 points (57-99) at critically ill department discharge. There was a significant improvement in FIM scale at the critically ill department discharge $(p<0.001)$. Patients that were discharged from the ICU had a lower median FIM scale when compared with the step-down unit and coronary unit $(80,54-99$ vs. $90,71-99$ vs. $54,39-64$ respectively; $p$ $<0.0001)$.

Conclusion: The FIM scale improved significantly during the critically ill department stay for patients accompanied by physiotherapists. Although at ICU discharge patients have a lower FIM scale when compared with the step-down unit and coronary unit, probably due to the short length of stay.
P28

Hospital mortality predictive factors following rapid response team activation in very old patients

Henrique Palomba*, Antonio Capone, Felipe M de T Piza, Michelle Jaures, Thiago D Corrêa

Hospital Israelita Albert Einstein, Morumbi, São Paulo, SP, Brazil

Critical Care 2015, 19(Suppl 2):P28; doi:10.1186/cc14681

Introduction: The number of very old ( $\geq 80$ years) patients with chronic illness and functional impairment requiring major medical attention is increasing, with great chances of adverse events during hospitalization. Rapid Response Teams (RRT) were implemented to improve the recognition and response to deteriorating ward patients; however, the characteristics and outcomes of very old patients assisted by RRT during hospitalization are not well described.

Objective: The primary objective was to evaluate the rate, characteristics and outcomes of very old patients seen by RRT during hospitalization.

Methods: A total of 538 very old patients assessed by RRT between January 2012 and December 2013 were included. Multivariate analysis was used to evaluate which variables were associated with hospital mortality. Early RRT call was defined as RRT activation $<48$ hours from hospital admission and late RRT call if it happened $>48$ hours from hospital admission.

Results: The mean age was $87 \pm 4.8$ years and $42 \%(n=224)$ were male. There were $193(36 \%)$ early calls and $345(64 \%)$ late calls. The main reasons for RRT activation were respiratory failure in $35 \%(n=186)$ and mental status changes in 15\% $(n=80)$. The distribution of RRT activation was uniform over the 24-hour period, with $55 \%(n=298)$ of calls during the day (7:00 a.m.-7:00 p.m.) and 45\% $(n=240)$ overnight (7:00 p.m.-7:00 a.m.). A total of 153 patients (28\%) were admitted to the ICU and the overall mortality rate was $20 \%(n=110)$. The multivariate analysis showed the following variables as significantly associated with hospital mortality: late ( $>48$ hours) RRT call (OR $1.73 ; 95 \% \mathrm{Cl} 1.07-2.79$ ), acute change in oximetry saturation to $<90 \%(\mathrm{OR} 1.56 ; 95 \% \mathrm{Cl} 1.01-2.40)$ and admission to step-down unit (OR 0.48; 95\% Cl 0.25-0.91).

Conclusion: In this study, hospital mortality predictive factors of very old patients seen by RRT during hospitalization were late RRT call and acute respiratory failure.

\section{P29}

Impact of obesity on critical care adult patients

Bruno F Mazza*, Claudia M Antunes, Fernando Costa, Lucas J Ricotta,

Luciano Quaini, Paulo F Marotto, Rosa GA Rocha, Samantha L de Almeida

Hospital Samaritano, Higienópolis, São Paulo, São Paulo, Brazil

Critical Care 2015, 19(Suppl 2):P29; doi:10.1186/cc14682

Introduction: Obesity is a growing problem in our society today. A great number of obese patients are admitted to the ICU, and there could probably be worse outcomes and more complications during their stay in the ICU.

Objective: The objective of this study is to analyze the epidemiological characteristics and the outcome of two groups of patients, one with a body mass index (BMI) $>30 \mathrm{~kg} / \mathrm{m}^{2}$ (group 1) and another group with BMI $<30 \mathrm{~kg} / \mathrm{m}^{2}$ (group 2).

Methods: A retrospective analysis was performed from January to December 2014, using the database EPIMED ${ }^{\otimes}$. We evaluated the epidemiological characteristics of patients and the outcome of them. Statistical analysis was performed using SPSS 22 software. The MannWhitney test was used to analyze numerical variables and the chi-square test to analyze the categorical variables. We considered data statistically significant if $p<0.05$.

Results: We evaluated 1478 patients, $327(22 \%)$ in the group with BMI $>30 \mathrm{~kg} / \mathrm{m} 2$ (G1) and 1151 (78 \%) in the group with (BMI) $<30 \mathrm{~kg} / \mathrm{m} 2$ (G2), who were admitted to the hospital ICU. The median of age in G1 was lower than G2 (60.4 \pm 17.5 vs. $65.4 \pm 20.5, p=0.001)$. There was no difference between the groups in length of stay in the critical care unit (7.0 \pm 12.0 vs. $5.8 \pm 8.1)$, in the hospital $(19.9 \pm 26.7$ vs. $17.7 \pm 25.6)$ and SAPS 3 score $(48.4 \pm 16.8$ vs. $47.5 \pm 16.3)(p=$ NS). There is no difference 
in the mortality ratio in the ICU (group 1: $5.1 \%$ vs. group 2: $6.8 \%, p=$ NS) or in the hospital (group 1: $11.9 \%$ vs. group 2: $10.1 \%, p=\mathrm{NS}$ ).

Conclusion: Obesity did not increase the mortality rate, or the ICU or the hospital length of stay. There was no difference in the gravity score between the groups. Current prognostic scoring systems do not include $\mathrm{BMI}$, possibly underestimating the risk of death, and other quality of care indexes in obese patients. New studies could be useful to clarify how BMI affects the management of obese patients in the ICU.

\section{P31}

Neck circumference as a complementary measure to identify risk of excess body mass in children younger than 2 years old

Daniela dos Santos, , Aila Aparecida Farias, Caroline Kroll,

Katherinne B Wanis Figueirêdo, Marco Fábio Mastroeni, Mayte Bertoli,

Sandra Ana Czarnobay, Silmara S de BS Mastroen

Universidade da Região de Joinville-Univille, Joinville, SC, Brazil

Critical Care 2015, 19(Suppl 2):P31; doi:10.1186/cc14683

Objective: To evaluate the effectiveness of neck circumference (NC) as a measure for assessing risk of excess body mass in children aged 13-24 months.

Methods: From a total of 435 children born in 2012 in a public maternity hospital of Joinville, Brazil, 279 participated in a new investigation 1 year later. Body mass, length and NC were collected in their homes between March 2013 and March 2014. The best cutoff value for identifying overweight/obese children using the body mass index (BMI) was determined by the receiver operating characteristic curve (ROC curve), according to gender and the age groups: 13-15 months, 16-19 months and 20-24 months.

Table 1(abstract cc14683) General characteristics of the children according to BMI category and sex, Joinville, SC, Brazil, 2013-2014

\begin{tabular}{llll}
\hline Characteristic & BMI $\leq$ P85 & BMI $>$ P85 & $\boldsymbol{p}$ value \\
\hline Boys & $(n=82)$ & $(n=60)$ & \\
Age (months) & $16.1(3.1)$ & $17.4(3.1)$ & 0.004 \\
Body mass $(\mathrm{kg})$ & $10,433.1(1124.7)$ & $12,839.7(1792.8)$ & $<0.001$ \\
Length $(\mathrm{cm})$ & $79.8(4.1)$ & $81.1(4.2)$ & 0.063 \\
BMl $\left(\mathrm{kg} / \mathrm{m}^{2}\right)$ & $16.3(1.0)$ & $19.4(1.4)$ & $<0.001$ \\
NC $(\mathrm{cm})^{\mathrm{a}}$ & $23.4(1.3)$ & $25.2(1.5)$ & $<0.001$ \\
Girls & $(n=81)$ & $(n=56)$ & \\
Age $(\mathrm{months})$ & $16.0(3.1)$ & $16.9(3.2)$ & 0.037 \\
Body mass $(\mathrm{kg})$ & $9834.5(1237.6)$ & $11,772.6(1524.7)$ & $<0.001$ \\
Length $(\mathrm{cm})$ & $78.4(4.2)$ & $78.7(4.3)$ & 0.443 \\
BMI $\left(\mathrm{kg} / \mathrm{m}^{2}\right)$ & $15.9(0.9)$ & $18.9(1.4)$ & $<0.001$ \\
NC $(\mathrm{cm})^{\mathrm{b}}$ & $22.9(1.3)$ & $24.7(1.5)$ & $<0.001$ \\
\hline
\end{tabular}

${ }^{\mathrm{a}} \mathrm{BMI} \leq \mathrm{P} 85, n=81 ; \mathrm{BMI}>\mathrm{P} 85, n=58$

${ }^{\mathrm{b}} \mathrm{BMI} \leq \mathrm{P} 85, n=80$

Table 2(abstract cc14683) Relationship between neck circumference (NC) and age, body mass, length and BMI by sex, Joinville, SC, Brazil, 2013-2014

\begin{tabular}{|c|c|c|c|c|}
\hline \multirow[t]{3}{*}{ Characteristic } & \multicolumn{4}{|l|}{ NC } \\
\hline & \multicolumn{2}{|l|}{ Boys } & \multicolumn{2}{|l|}{ Girls } \\
\hline & rho & $p$ value & rho & $p$ value \\
\hline Age & 0.024 & 0.778 & 0.124 & 0.151 \\
\hline Body mass & 0.520 & $<0.001$ & 0.660 & $<0.001$ \\
\hline Length & 0.117 & 0.172 & 0.358 & $<0.001$ \\
\hline BMI & 0.635 & $<0.001$ & 0.606 & $<0.001$ \\
\hline
\end{tabular}

Table 3(abstract cc14683) Spearman correlation coefficients (rho) of the relation between BMI and neck circumference (NC) by age and sex, Joinville, SC, Brazil, 2013-2014

\begin{tabular}{llll}
\hline Age (months) & $\boldsymbol{n}$ & \multicolumn{2}{l}{ BMI-NC } \\
\cline { 3 - 4 } & & $\boldsymbol{r h o}$ & $\boldsymbol{p}$ value \\
\hline Boys & 65 & 0.604 & $<0.001$ \\
$13-15$ & 44 & 0.663 & $<0.001$ \\
$16-19$ & 30 & 0.719 & $<0.001$ \\
$20-24$ & & & \\
Girls & 73 & 0.642 & $<0.001$ \\
$13-15$ & 30 & 0.419 & 0.021 \\
$16-19$ & 33 & 0.604 & $<0.001$ \\
$20-24$ & & & \\
\hline
\end{tabular}

Table 4(abstract cc14683) Area under the curve (AUC), frequency $(n)$, optimal cutoff points, sensitivities and specificities for neck circumference (NC) associated with excess body mass in three age groups of boys and girls, Joinville, SC, Brazil, 2013-2014

\begin{tabular}{llllll}
\hline $\begin{array}{l}\text { Age } \\
\text { (months) }\end{array}$ & $n$ & AUC $(\mathbf{9 5} \% \mathrm{Cl})$ & Cutoff & $\begin{array}{l}\text { Sensitivity } \\
(\%)\end{array}$ & $\begin{array}{l}\text { Specificity } \\
(\%)\end{array}$ \\
\hline $\begin{array}{l}\text { Boys } \\
13-15\end{array}$ & 65 & $0.796(0.683-0.909)$ & 23.6 & 85.0 & 48.9 \\
$16-19$ & 44 & $0.876(0.771-0.981)$ & 23.9 & 81.8 & 63.6 \\
$20-24$ & 30 & $0.902(0.000-1.000)$ & 24.0 & 75.0 & 92.9 \\
Girls & & & & & \\
$13-15$ & 73 & $0.814(0.709-0.919)$ & 23.4 & 73.1 & 61.7 \\
$16-19$ & 30 & $0.697(0.509-0.885)$ & 23.5 & 76.9 & 52.9 \\
$20-24$ & 33 & $0.796(0.644-0.947)$ & 23.6 & 76.5 & 68.7 \\
\hline
\end{tabular}

$\mathrm{Cl}$ confidence interval

Results: NC was positive and significant. The NC cutoff points increased with increasing age group in boys $(23.6,23.9$ and $24.0 \mathrm{~cm})$ and girls $(23.4$, 23.5 and $23.6 \mathrm{~cm}$ ), respectively, for the 13-15, 16-19 and 20-24 age groups. See Tables 1, 2, 3, 4.

Conclusion: Our results suggest that NC can also be used to screen risk of excess body mass and upper fat distribution in children aged 13-24 months. However, further studies with a larger sample in order to complement our data will be required.

P32

Organizational characteristics, outcomes and resource use in ICUs: the ORCHESTRA Study

Derek C Angus ${ }^{1 *}$, Fernando Bozza ${ }^{2}$, Jeremy Kahn ${ }^{1}$, Jorge Salluh², Marcio Soares², ORCHESTRA Study Investigators's, Pedro Brasil ${ }^{2}$

'University of Pittsburgh Medical Center, Pittsburgh, PA, USA; ${ }^{2}$ D'Or Institute for Research and Education, Botafogo, Rio de Janeiro, RJ, Brazil Critical Care 2015, 19(Suppl 2):P32; doi:10.1186/cc14684

Introduction: Detailed information on organizational factors in ICUs located in emerging countries is scarce.

Objective: To investigate the impact of organizational factors on patient outcomes and resource use in a large sample of Brazilian ICUs.

Methods: Retrospective cohort study of 59,483 patients (medical admissions: $39,734(67 \%)$ ) admitted to 78 ICUs (private hospitals, $n=67$ (86\%); medical-surgical; $n=62(79 \%)$ ) during 2013. We retrieved demographic, clinical and outcome data from an electronic ICU quality registry (Epimed Monitor System). We surveyed ICUs using a standardized questionnaire regarding organizational aspects, staffing patterns and 
process of care. We used multilevel logistic regression analysis to identify factors associated with hospital mortality. Efficient resource use was assessed by estimating standardized mortality rates (SMR) and standardized resource use (SRU) adjusted for the SAPS 3 score.

Results: Forty (51\%) ICUs had critical care training programs. The median nurse-bed ratio was $0.20(\mathrm{IQR}, 0.15-0.28)$ and board-certified intensivists were present $24 / 7$ in $16(21 \%)$ of the ICUs. Routine clinical rounds occurred in 67 (86\%) and daily checklists were used in 36 (46\%) ICUs. The most frequently implemented protocols focused on sepsis management and VAP and CLABSI prevention. Median number of patients per center was 898 (585-1715). SAPS 3 score was 41 (33-52) points. ICU and hospital mortality rates were $9.6 \%$ and $14.3 \%$. Adjusting for relevant patients' characteristics (SAPS 3 score, admission diagnosis, chronic health status, comorbidities, MV use), case volume and type of $\mathrm{ICU}$, clinical protocols jointly managed by different care providers $(\mathrm{OR}=$ $0.23(95 \% \mathrm{Cl}, 0.08-0.64), p=0.005)$ were associated with lower mortality. Estimated SMR and SRU were 0.97 (0.72-1.15) and 1.06 (0.89-1.37). There were 28 (36\%) "most efficient" (ICUs with both SMR and SRU median), 11 (14\%) "overachieving" (ICUs with low SMR and high SRU) and 11 (14\%) "underachieving" (ICUs with high SMR and low SRU) ICUs. "Most efficient" ICUs were usually located in private hospitals, with step-down units and training programs in critical care. In univariate analyses comparing "most efficient" and "least efficient" ICUs, a graduated nurse-bed ratio $>0.25$ $(\mathrm{OR}=4.40(1.04-18.60))$ was associated with efficient resource use. Daily checklists also tended to be associated with efficient resource use $(\mathrm{OR}=$ $2.89(0.95-8.72), p=0.057)$.

Conclusion: In emerging countries such as Brazil, organizational factors are potential targets to improve patient outcomes and efficient resources in ICUs.

Acknowledgements: Funded by CNPq and FAPERJ. Endorsed by BRICNet.

\section{P33}

Outcomes in critically ill patients with cancer-related complications Viviane BL Torres ${ }^{1 *}$, Juliana Vassalo ${ }^{1}$, Nelson Spector ${ }^{1}$, Fernando A Bozza ${ }^{2}$, Jorge IF Salluh 1,2,3, Marcio Soares ${ }^{1,2,3}$

${ }^{1}$ Postgraduate Program in Internal Medicine, School of Medicine, UFRJ, Rio de Janeiro, RJ, Brazil; ${ }^{2}$ IDOR-D'Or Institute for Research and Education, Rio de Janeiro, RJ, Brazil; ${ }^{3}$ Postgraduate Program, Instituto Nacional de Câncer, Rio de Janeiro, RE, Brazil

Critical Care 2015, 19(Suppl 2):P33; doi:10.1186/cc14685

Introduction: Cancer patients are at risk for severe complications related to the underlying malignancy or its treatment, and therefore usually require admission to ICUs.

Objective: To evaluate the clinical characteristics and outcomes in this subgroup of patients.

Methods: Analysis of two prospective cohorts of cancer patients admitted to ICUs. We used multivariable logistic regression to identify variables associated with hospital mortality.

Results: Out of 2028 patients, 456 (23 \%) had cancer-related complications. Compared with those without complications, they more frequently had worse performance status (PS) $(57 \%$ vs. $36 \%$ with PS $\geq 2$ ), active malignancy ( $43 \%$ vs. $5 \%$ ), need for vasopressors ( $45 \%$ vs. $34 \%$ ), mechanical ventilation (70\% vs. $51 \%$ ) and dialysis (12\% vs. $8 \%)(P<0.001$ for all analyses). ICU (47 \% vs. $27 \%)$ and hospital (63\% vs. $38 \%)$ mortality rates were also higher in patients with complications $(P<0.001)$. Chemo/radiation therapy-induced toxicity $(6 \%)$, venous thromboembolism $(5 \%)$, respiratory failure $(4 \%)$, gastrointestinal involvement (3\%) and vena cava syndrome (VCS) $(2 \%)$ were the most frequent complications. In multivariable analysis, the presence of cancer-related complications per se was not associated with mortality (odds ratio $(\mathrm{OR})=1.25(95 \%$ confidence interval, 0.94-1.66)). However, among the individual complications, VCS $(O R=3.79(1.11-12.92))$, gastrointestinal involvement $(\mathrm{OR}=3.05(1.57-5.91))$ and respiratory failure $(O R=1.96(1.04-3.71))$ were independently associated with worse outcomes.

Conclusion: The prognostic impact of cancer-related complications was variable. Although some complications were associated with worse outcomes, the presence of a severe acute cancer-related complication per se should not guide decisions to admit a patient to the ICU.
P34

Predictive factors of in-hospital mortality in very old patients following rapid response team activation

Henrique Palomba*, Andréia Pardini, Antonio Capone Neto,

Felipe M de T Piza, Michele Jaures, Thiago D Corrêa

Hospital Israelita Albert Einstein, Morumbi, São Paulo, SP, Brazil

Critical Care 2015, 19(Suppl 2):P34; doi:10.1186/cc14686

Introduction: The number of very old patients ( 80 years old) with chronic illness and functional impairment requiring major medical attention is increasing. Rapid Response Teams (RRT) were implemented to improve the recognition and response to deteriorating ward patients. However, the characteristics and outcomes of very old patients assisted by RRT during hospitalization are not well described.

Objective: The primary objective was to evaluate the rate, characteristics and outcomes of very old patients ( 80 years old) assisted by RRT during hospitalization.

Methods: A total of 538 very old patients assessed by RRT between January 2012 and December 2013 was included in this analysis. A multivariate logistic regression analysis was undertaken to address which predictors were associated with increased in-hospital mortality. Early RRT call was defined as RRT activation within 48 hours from hospital admission and late RRT call if it happened afterwards.

Results: From January 2012 and December 2013, 2072 patients were assisted by the RRT. Very old patients (age $\geq 80$ years) accounted for $26 \%$ $(538 / 2072)$ of patients. The mean (SD) age was 87 (4.8) years and $42 \%$ $(n=224)$ were male. There were $193(36 \%)$ early RRT calls and 345 (64 $\%)$ late RRT calls. The main reasons for RRT activation were respiratory failure in $35 \%(n=186)$ and mental status changes in $15 \%(n=80)$. The distribution of RRT activation was uniform over the 24-hour period, with $55 \%(n=298)$ of calls during the day (7:00 a.m.-7:00 p.m.) and $45 \%(n=$ 240) overnight (7:00 p.m.-7:00 a.m.). A total of 153 patients ( $28 \%$ ) were admitted to the ICU and the overall mortality rate was $21.5 \%(110 / 511)$. Late RRT call (OR 1.73; $95 \% \mathrm{Cl} 1.07-2.80 ; p=0.025)$ and acute changes in peripheral oxygen saturation below $90 \%$ (OR 1.56; $95 \% \mathrm{Cl} 1.01-2.40 ; p=$ 0.044 ) were associated with increased risk of in-hospital death while admission to a step-down unit (OR 0.49; $95 \% \mathrm{Cl} 0.26-0.92 ; p=0.026)$ was associated with a decreased risk of in-hospital death.

Conclusion: Multiple factors relating to the nature of RRT activation and very old patient characteristics are associated with in-hospital mortality. This information may be useful for risk stratification and determination of an appropriate treatment strategy for very old patients during hospitalization.

P35

Reduce and optimize critical care unit noise

Ana Maria Cavalheiro*, Carolina L Pires, Fabiana Aparecida Sarmento, Leonardo José R Ferraz, Neide M Lucino

Hospital Albert Einstein, Morumbi, São Paulo, SP, Brazil

Critical Care 2015, 19(Suppl 2):P35; doi:10.1186/cc14687

Introduction: Noise is an environmental stressor that is known to have physiological and psychological effects. The body responds to noise in the same way as it responds to stress and over time can impair health. Research clearly shows that hospital noise levels exceed noise level recommendations and have the potential to increase complications in patients. What is less known is the effect Critical Care Unit noise has on nurses.

Objective: The purpose of this article is to discuss Critical Care Unit noise. Methods: By measuring noise during peak and off-peak ICU hours, analysts can detect and correct the noise. Noise meter readings established by a pollution control board offer quantitative readings that help predict, and therefore prevent, future noise problems. We identified the primary sources for noise pollution through a questionnaire applied to these patients and caregivers. Complaints related to undesirable noises were alarms, parametric monitors, and noise caused by the companions of other patients and the multidisciplinary team. Noise measurements were carried out by occupational medicine, which identified the noise peaks as 8:00 p.m. 67 dB, 9:00 a.m. $62 \mathrm{~dB}$ and 1:00 p.m. 63.8 dB. The Deming model was adopted and by brainstorming with the multidisciplinary team we decided to use educational strategies, lectures for the multidisciplinary 
team and bookmark images stimulating silence for companions in the ICU. The lectures to staff were conducted by psychologists and speech therapists about using the voice and care related to behavior and education. We made new measures of noise after 30 days and noticed a significant drop to $45 \mathrm{db}$ in the night period, $54 \mathrm{~dB}$ in the morning and $53 \mathrm{db}$ in the afternoon.

Conclusion: Educational activities within work are still the best way to obtain significant results for healthy behaviors to ensure the safety of patients and professionals.

P39

Assessment of fluid responsiveness in spontaneously breathing critically ill patients

Renato CF Chaves ${ }^{1 *}$, Murillo SC Assunção ${ }^{2}$

'Department of Intensive Care Medicine, Hospital Santa Lúcia, Brasília, DF, Brazil; ${ }^{2}$ Department of Intensive Care Medicine, Hospital Israelita Albert

Einstein, São Paulo, SP, Brazil

Critical Care 2015, 19(Suppl 2):P39; doi:10.1186/cc14688

Introduction: One of the main challenges in critical patient management is to assess the blood volume and determine which patients will benefit from volume expansion and which patients will benefit from support with vasopressor and/or inotropic drugs. It is known that $40-72 \%$ of critical patients respond to volume expansion with increased stroke volume or cardiac index.

Objective: To search the literature for methods assessing fluid responsiveness in spontaneously breathing critically ill patients.

Methods: The present study is a systematic literature review. We searched randomized clinical trials through a blind search performed by two independent authors in any language in the National Library of Medicine from 2009 to 2014.

Results: We selected three articles for full review and analysis, totaling 116 patients. The results are shown in Table 1.

Conclusion: This systematic review supports the beneficial effects of adopting maneuvers that amplify the hemodynamic changes, increasing the accuracy of methods to predict fluid responsiveness in spontaneously breathing critically ill patients.

References

1. Dong ZZ, Fang $Q$, Zheng $X$, Shi H: Passive leg raising as an indicator of fluid responsiveness in patients with severe sepsis. World J Emerg Med 2012, 3:191-6.

2. Préau S, Dewavrin F, Soland V, Bortolotti P, Colling D, Chagnon JL, Durocher A, Saulnier F: Hemodynamic changes during a deep inspiration maneuver predict fluid responsiveness in spontaneously breathing patients. Cardiol Res Pract 2012, 2012:191807.

3. Hong DM, Lee JM, Seo JH, Min JJ, Jeon Y, Bahk JH: Pulse pressure variation to predict fluid responsiveness in spontaneously breathing patients: tidal vs. forced inspiratory breathing. Anaesthesia 2014, 69:717-22.

4. Préau S, Saulnier F, F Dewavrin, Durocher A, Chagnon JL: Passive leg raising is predictive of fluid responsiveness in spontaneously breathing patients with severe sepsis or acute pancreatitis. Critical Care Medicine 2010, 38(3):819-825.
P43

Could saline instillation displace bacteria from the endotracheal tube biofilm to lower airways during suctioning procedure?

Anália Cristina CR Franchi", Antônio Pazin Filho, Camila Bottura,

Carlos Henrique Miranda, Fernanda B Ferreira, Marcos de C Borges,

Rodrigo de C Santana, Valéria T Okino

Division of Emergency Medicine, Ribeirão Preto School of Medicine, São

Paulo University, Centro, Ribeirão Preto, SP, Brazil

Critical Care 2015, 19(Suppl 2):P43; doi:10.1186/cc14689

Introduction: A biofilm is found on the inner side of endotracheal tubes (ETT) in mechanically ventilated patients. Saline instillation inside the ETT during the suctioning procedure is very common. This procedure could displace bacteria to the lower airways, increasing the risk of ventilatorassociated pneumonia (VAP).

Objective: Evaluation of the bacteriological cultures of the ETT lavage with saline after extubation of mechanically ventilated patients to verify dislocation of bacteria through this procedure.

Methods: The ETT was removed using an aseptic technique during extubation. Saline $(10 \mathrm{ml})$ was instilled into the tube, and the drainage fluid was collected on the other side. This material was sent to microbiological cultures in two different culture mediums (chocolate blood agar and MacConkey). We considered the quantitative culture of more than 100,000 $\mathrm{UFC} / \mathrm{ml}$ as positive. The characteristics of the patients with and without positive cultures were compared.

Results: Forty endotracheal tubes were analyzed $(n=40)$. Positive culture was observed in eight tubes ( $20 \%)$. The bacteria observed were: five Gram-positive (Staphylococcus aureus in three, Streptococcus pneumoniae in one and Staphylococcus haemolytics in one) and three Gram-negative (Acinetobacter baumani, Klebsiella pneumoniae, Enterobacter cloacae). We did not observe differences between the group with positive and negative cultures in relation to demographic and clinical characteristics, intubation time and tube diameter $(p>0.05)$.

Conclusion: The use of saline during an endotracheal suctioning procedure can dislocate pathogenic bacteria from the endotracheal tube biofilm to the lower airways, and could increase the risk of VAP. The use of saline should be minimized during patient care.

\section{P44}

Success of a urinary catheter insertion team in reducing urinary infections in the ICU

Deianira Regagnin*, Debora Schettini da Silva Alves, Luciana Reis Guastelli Department of Critically III Patients, Hospital Israelita Albert Einstein, São Paulo, SP, Brazil

Critical Care 2015, 19(Suppl 2):P44; doi:10.1186/cc14690

Introduction: About 8-21 \% of hospital infections in ICUs are urinary [1,2] $80 \%$ of them being associated with the use of urinary catheters [3] Several studies show that the early removal of urinary catheters reduces the rate of urinary tract infection. However, critically ill patients who require this device do not have the option to remove. For this group, the best preventive measure seems to be educative activity for the nursing staff responsible for the insertion and manipulation of this device.

Table 1(abstract cc14688) Accuracy of hemodynamic parameters for predicting fluid responsiveness

\begin{tabular}{|c|c|c|c|c|c|c|c|c|c|}
\hline \multirow[b]{2}{*}{ Parameter } & \multicolumn{3}{|c|}{ Préau et al., $2010[4](n=34)$} & \multicolumn{4}{|c|}{ Préau et al., $2012[2](n=23)$} & \multicolumn{2}{|c|}{ Hong et al., $2014[3](n=59$} \\
\hline & $\Delta$ SV-PLR & $\triangle \mathrm{PP}-\mathrm{PLR}$ & $\Delta$ VF-PLR & $\Delta \mathrm{PP}$ & $\Delta$ PP-dim & $\Delta \mathrm{VF}$ & $\Delta$ VF-dim & $\triangle \mathrm{PP}-\mathrm{TB}$ & $\triangle \mathrm{PP}-\mathrm{FB}$ \\
\hline Threshold value (\%) & 10 & 9 & 8 & 10 & 12 & 10 & 12 & NS & 13.7 \\
\hline Sensitivity (\%) & 86 & 79 & 86 & 60 & 90 & 60 & 90 & NS & 89.7 \\
\hline Specificity (\%) & 90 & 85 & 80 & 100 & 100 & 100 & 100 & NS & 86.7 \\
\hline ROC curve area & 0.94 & 0.86 & 0.93 & 0.71 & 0.95 & 0.74 & 0.95 & 0.618 & 0.910 \\
\hline$p$ value & $<0.001$ & $<0.01$ & $<0.001$ & 0.02 & $<0.01$ & 0.02 & $<0.01$ & 0.112 & $<0.0001$ \\
\hline Responders & 14 & 14 & 14 & 10 & 10 & 10 & 10 & 29 & 29 \\
\hline
\end{tabular}

Dim deep inspiration maneuver-induced change, $F B$ forced inspiratory breathing, NS non specified, $P L R$ passive leg raising induced change, $\triangle P P$ pulse pressure, $\triangle S V$ stroke volume, $T B$ tidal breathing, $\triangle V F$ peak velocity of femoral artery flow 
Objective: To create a team of professionals trained in the insertion of urinary catheters and to organize actions aimed at reducing the rate of urinary tract infection associated with urinary catheters in the ICU.

Methods: Prospective study conducted for 12 months in the ICU. Started in July 2013, the intervention program involved the creation of a qualified team for the insertion of urinary catheter and the creation of audits to stimulate the removal of inappropriate urinary catheters and assess the process of inserting these devices. The obtained results were compared with the 12 months preceding the beginning of the interventions.
Results: Comparison between August 2012-July 2013 and August 2013July 2014 (Table 1, Figures 1 and 2) shows that there was a fall of $57.2 \%$ (2.4-1.0, $p=0.040)$ in the rate of urinary tract infection associated with a urinary catheter and a reduction of $13.4 \%$ (from 0.24 to $0.21, p=0.001$ ) in the utilization rate of urinary catheters. In the 12 months after intervention (August 2013-July 2014) the percentage of compliance of technical insertion of urinary catheter was $97 \%$ and the inappropriate removal rate of urinary catheters was $85 \%$ (Table 2).

Conclusion: The results show that low-cost educational interventions can reduce urinary infections and provide more security for patients in ICUs.

Table 1(abstract cc14690) Incidence density ratio urinary tract infection and utilization ratio urinary catheter before and after interventions according to the location

\begin{tabular}{|c|c|c|c|c|c|c|c|c|c|}
\hline Place & Ratio & Time & Mean & DP & Median & Min & Max & Mean reduction (\%) & $p$ value \\
\hline \multirow[t]{4}{*}{$\mathrm{ICU}$} & IDR UTI & Before & 1.69 & 1.47 & 1.57 & 0.00 & 4.76 & 40.9 & 0.286 \\
\hline & & After & 1.00 & 1.62 & 0.00 & 0.00 & 4.66 & & \\
\hline & UR & Before & 0.57 & 0.05 & 0.57 & 0.47 & 0.62 & 11.3 & 0.016 \\
\hline & & After & 0.50 & 0.07 & 0.54 & 0.37 & 0.57 & & \\
\hline \multirow[t]{4}{*}{ SDU } & IDR UTI & Before & 4.01 & 4.52 & 3.38 & 0.00 & 12.71 & 66.9 & 0.084 \\
\hline & & After & 1.33 & 2.45 & 0.00 & 0.00 & 6.37 & & \\
\hline & UR & Before & 0.11 & 0.02 & 0.11 & 0.09 & 0.13 & 25.0 & $<0.001$ \\
\hline & & After & 0.08 & 0.01 & 0.08 & 0.06 & 0.09 & & \\
\hline \multirow[t]{4}{*}{$I C U+S D U$} & IDR UTI & Before & 2.39 & 1.89 & 2.08 & 0.00 & 6.59 & 57.2 & 0.040 \\
\hline & & After & 1.02 & 1.07 & 1.20 & 0.00 & 3.23 & & \\
\hline & UR & Before & 0.24 & 0.02 & 0.25 & 0.21 & 0.26 & 13.4 & 0.001 \\
\hline & & After & 0.21 & 0.02 & 0.22 & 0.16 & 0.23 & & \\
\hline
\end{tabular}

Student $t$ test

$S D U$ step down unit, IDR UTI incidence density ratio of urinary tract infection,

$U R$ utilization ratio of urinary catheter

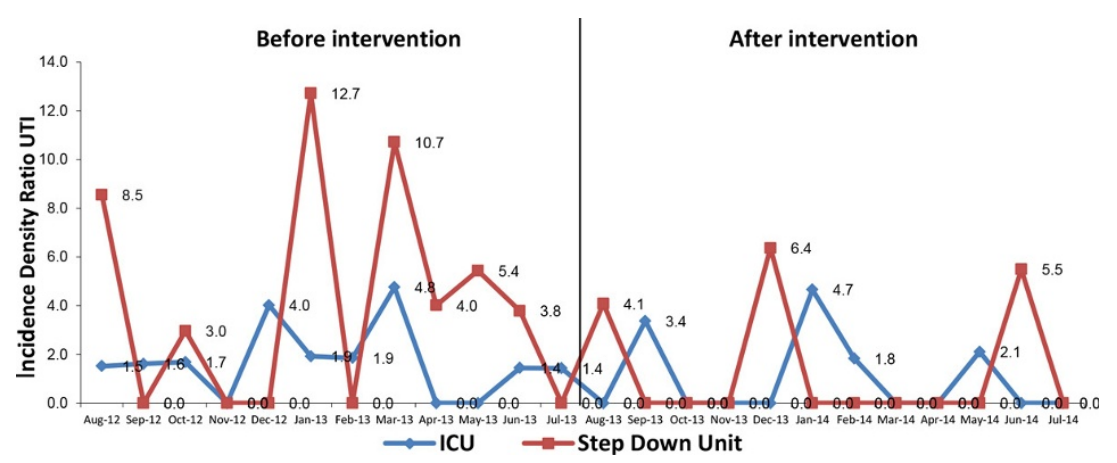

Figure 1(abstract cc14690) Incidence density ratio of urinary tract infections before and after interventions

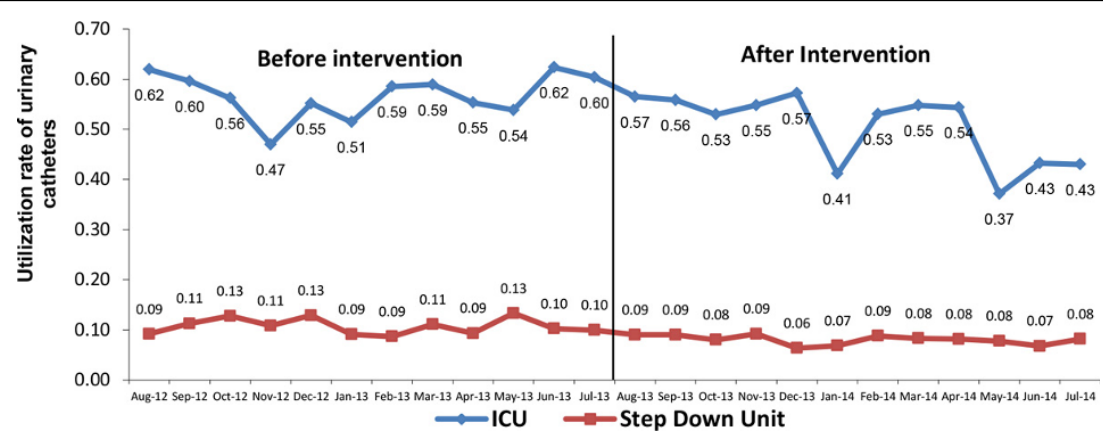

Figure 2(abstract cc14690) Utilization rate of urinary catheters before and after interventions 


\section{Table 2(abstract cc14690) Results of audited items between August 2013 and July 2014}

\begin{tabular}{llll}
\hline & ICU & SDU & ICU + SDU \\
\hline Number of patients observed & 9571 & 25,716 & 35,287 \\
Number of patients with IDC & 4752 & 2370 & 7122 \\
$\%$ Urinary catheter appropriate & 92 & 86 & 90 \\
$\%$ Urinary catheter inappropriate & 10 & 16 & 12 \\
\% Discontinued inappropriate IDC & 97 & 70 & 85 \\
Number of insertions of IDC audited & 803 & 280 & 1083 \\
\% Proper techniques for insertion of IDCs & 99 & 95 & 97 \\
\hline
\end{tabular}

IDC indwelling urinary catheters; SDU step down unit

\section{References}

1. Eriksen HM, Iversen BG, Aavitsland P: Prevalence of nosocomial infections in hospitals in Norway, in 2002 and 2003. J Hosp Infect 2005, 60: 40-5.

2. Lizioli A, Privitera G, Alliata E, Antonietta Banfi IN, Boselli L, Panceri ML, et al: Prevalence of nosocomial infections in Italy: result from the Lombardy survey in 2000. J Hosp Infect 2003, 54:141-8.

3. Klevens RM, Edward JR, Richards CL Jr, et al: Estimating health care associated infections and Deaths in US hospitals. Public Health Rep 2007 122:160e.

\section{P48}

Does a specialized neurological ICU have better performance when compared with a general ICU?

Bruno F Mazza*, Maria Fernanda Z Gatti, Rita de Cássia R de Macedo,

Rosa GA Rocha, Samantha L Almeida

Hospital Samaritano, Higienópolis, São Paulo, SP, Brazil

Critical Care 2015, 19(Suppl 2):P48; doi:10.1186/cc14691

Introduction: The costs of ICUs are high. American data show that they represent about $13 \%$ of hospital costs, $4 \%$ of the amount allocated to national health and $0.66 \%$ of US GDP. Thus, it is necessary to use the best possible resource seeking the best for the institution and for patients. Studies show that a specialized unit in the treatment of neurological patients (ICU-N) has better results when compared with general ICUs (ICU-G).

Objective: The objective of this study is to compare the results of patients with hospitalization for clinical neurological disorders in a neurological ICU with patients with the same diseases in general units.

Methods: A retrospective analysis was performed from June to December 2014, using the database EPIMED ${ }^{\circledR}$. All patients who were hospitalized with primary and secondary diagnoses of neurologic disorders were evaluated. SAPS severity score, length of stay and outcomes, and epidemiological profiles were analyzed. The results were descriptive and the percentage of cases, mean and standard deviation in the groups were analyzed.

Results: Neurological disorders accounted for $17 \%$ of admissions to the general units, against $94 \%$ in the specialized unit. The mean age (ICU-N: $65 \pm 15.16$ vs. ICU-G: $63 \pm 20.28$ ) and sex (ICU-N: $45 \%$ vs. ICU-G: $50 \%$ male) were similar in both units. The most common diseases in the units were ischemic stroke, seizures, subarachnoid hemorrhage, intracerebral hemorrhage and transient ischemic attack. The average length of stay in the unit $(7.29 \pm 9.60$ vs. $12.56 \pm 21.22$ days) and hospital (ICU-N: $14.39 \pm$ 24.21 vs. ICU-G: $22.11 \pm 43,12$ days) was lower in the ICU-N, and $78 \%$ of patients had shorter hospital stays than 7 days, compared with $67 \%$ in the ICU-G. The severity as measured by the SAPS 3 score was greater in ICU-G compared with the ICU-N $(52.81 \pm 16.05$ vs. $49.53 \pm 10.74)$ with a higher expected probability of death (26 $68 \%$ vs. $20.01 \%$ ). On the other hand, the ICU-N hospital mortality observed (6.52\% vs. $33.58 \%)$ and thus the standardized mortality rate $(0.33$ vs. 1.36$)$ was lower.

Conclusion: The data relating to gender, age and diseases are similar in both units. The severity of disease is higher in the ICU-G. However, when analyzing the length of stay and mortality, the specialized unit has better performance, reducing the length of stay in the ICU and in the hospital and showing a significant difference in mortality.
P50

Brain death and changes in solid organ characteristics due to acute lack of female sex hormones

Roberto Armstrong Junior, Ana Cristina B Faloppa, Guilherme K Kudo,

Luiz Felipe P Moreira, Paulina Sannomiya, Raif R Simão, Sueli G Ferreira

Heart Institute (INCOR), University of São Paulo Medical School, São Paulo,

SP, Brazil

Critical Care 2015, 19(Suppl 2):P50; doi:10.1186/cc14692

Introduction: Previous studies have suggested that female sex hormones have a protective action, because they contribute to reduce the inflammatory degree in females after trauma.

Objective: This study aimed to investigate sex differences in the course of the inflammatory process in rats subjected to brain death (BD).

Methods: Wistar rats were randomized into three groups (male rats, $n=5$; female rats, $n=10$; and ovariectomized rats, $n=5$ ) and subjected to BD by rapid inflation of a catheter Fogarty ${ }^{\circledast} 4 \mathrm{~F}$. The liver, kidneys, lungs and the heart were collected after 6 hours and samples $(4 \mu \mathrm{m})$ were stained with $\mathrm{H} \& \mathrm{E}$ for histological analyses. Leukocyte infiltration, edema and hemorrhage were measured and data were compared using GraphPad Prism v.6.10, and $p$ values lower than 0.05 were considered significant.

Results: Female rats exhibited increased leukocyte infiltration into the lungs and the heart when compared with male rats $(p=0.009$ in the lungs and $p$ $=0.022$ in the heart) and presented also a sudden decrease in estradiol levels 6 hours after BD $(p=0.01)$. The intensity of hemorrhage was greater in ovariectomized rats compared with the other groups $(p=0.001)$ in the lungs. All groups presented slight to moderate leukocyte infiltration and absence to slight hemorrhage in the liver. Leukocyte infiltration had a wide distribution in female rat kidneys, and in male and ovariectomized rat kidneys infiltration varied from absent to slight.

Conclusion: The increased inflammation in the lungs and heart of female rats might be a result of the lack of female sex hormones. Therefore, the idea of introducing a therapeutic use of female sex hormones on female BD donors could be considered.

Acknowledgements: Financial support: FAPESP 2013/20282-0

P53

Interference of blood pressure control within 24 hours in acute ischemic stroke: systematic review protocol

Arnaldo A da Silva*, Alvaro N Atallah, Gisele S Silva, Gustavo José M Porfírio Universidade Federal de São Paulo, Vila Clementino, São Paulo, SP, Brazil Critical Care 2015, 19(Suppl 2):P53; doi:10.1186/cc14693

Stroke is the third most common cause of death in most industrialized countries, with an estimated global mortality of 4.7 million yearly. A stroke occurs every 53 seconds in North America and by 2002 was projected to become the fourth leading burden of disease worldwide. Stroke killed 283,000 people in 2000 and accounted for about one in every 14 deaths in the United States. Each year, about 700,000 people suffer a new or recurrent stroke. It is the major cause of serious, long-term disability, with more than $1,100,000$ American adults reporting functional limitations resulting from stroke.

Review of the evidence on how acute variation in blood pressure (BP) during the first 24 hours of acute ischemic stroke can influence outcome, considering interesting preliminary evidence that without intervening medications may be superior to some use of drugs in modifying an acute rise in BP, and suggesting that the blood pressure decline spontaneously without administration of medication may also have an influence on the acquired disabilities.

We searched the Cochrane Central Register of Controlled Trials (CENTRAL) (The Cochrane Library 2013, Issue 12), MEDLINE (1954-July 2013), EMBASE (1980-July 2013), CINAHL (1982-July 2013), Database of Research in Stroke (DORIS) (2008-2013), Latin American and Caribbean Health Sciences Literature (LILACS) (1982-December 2013) and reference lists of articles. We contacted researchers in the field. We did a grey literature search for articles published until July 2013. We also searched Dissertation Abstracts International via Dissertation Express, and the metaRegister of Controlled Trials. In an effort to identify further published, unpublished and ongoing trials, we searched ongoing trials registers and SCOPUS.

Inclusion criteria: 1 , age 18-75 years; 2 , clinical signs consistent with the diagnosis of ischemic stroke; 3, treatment onset within 3-9 hours after 
stroke onset; 4, no prior neurologic event that would obscure the interpretation of the signal and current presenting neurologic deficits (modified Rankin scale $=1$ ); 5 , National Institutes of Health Stroke Scale (NIHSS) score $>4$ and at least moderate limb weakness; 6 , MRI screening to be started within 7.5 hours after stroke onset; 7 , perfusion abnormality of $>2 \mathrm{~cm}$ in diameter involving hemispheric gray matter; 8 , perfusion/ diffusion mismatch of 20; 9, magnetic resonance angiography shows TICI grade 0 or 1. Exclusion criteria: 1, prestroke score on the modified Rankin Scale $>2$ or on the Barthel Index.

Review authors will work independently to assess risk of bias using criteria described in the Cochrane Handbook for Systematic Reviews of Interventions (Higgins 2011) to assess trial quality. This set of criteria is based on evidence of associations between overestimate of effect and high risk of bias of the article such as sequence generation, allocation concealment, blinding, incomplete outcome data and selective reporting. If the raters disagreed, the final rating was made by consensus, with the involvement of another member of the review group. Where inadequate details of randomization and other characteristics of trials were provided, authors of the studies were contacted in order to obtain further information. Nonconcurrence in quality assessment was reported, but if disputes arose as to in which category a trial was to be allocated, again, resolution was made by discussion. The level of risk of bias was noted in both the text of the review and in the "Summary of findings tables". Primary outcomes: death or dependency at the end of scheduled follow-up. Dependency is defined as being severely dependent on others in activities of daily living, or being significantly disabled; this corresponds to a Barthel Index score or a modified Rankin Scale grade 3-6 at 3-month follow-up. Secondary outcomes: 1, standardized nondisease-specific instrument for describing and valuating health-related quality of life; EQ-5D (EuroQol) questionnaire. 2, NIHSS measure of neurologic deficit; the Barthel Index measure of activities of daily living; the Modified Rankin Scale measure of the degree of disability or dependence in daily activities for 90 days followup. 3, average time of hospital discharge. 4, time to discharge from the neuro ICU or neuro critical care unit. 5, assessment of systolic and diastolic blood pressure control. 6, causality assessment of adverse events following blood pressure reduction within 24 hours of acute ischemic stroke.

\section{References}

1. Berkhemer $O A$, Fransen $P S$, Beumer D, et al: A randomized trial of intraarterial treatment for acute ischemic stroke. N Engl J Med 2015, 372:121-20.

2. Adams HP Jr, del Zoppo G, Alberts MJ, Bhatt DL, Brass L, Furlan A, et al:

Guidelines for the early management of adults with ischemic stroke: a guideline from the American Heart Association/American Stroke Association Stroke Council, Clinical Cardiology Council, Cardiovascular Radiology and Intervention Council, and the Atherosclerotic Peripheral Vascular Disease and Quality of Care Outcomes in Research Interdisciplinary Working Groups: the American Academy of Neurology affirms the value of this guideline as an educational tool for neurologists. Stroke 2007, 38:1655-711.

3. Adams HP Jr, Davis PH, Leira EC, Chang KC, Bendixen BH, Clarke WR, et al: Baseline NIH Stroke Scale score strongly predicts outcome after stroke: a report of the Trial of Org 10172 in Acute Stroke Treatment (TOAST). Neurology 1999, 53:126-31.

4. Tissue plasminogen activator for acute ischemic stroke. The National Institute of Neurological Disorders and Stroke rt-PA Stroke Study Group. N Engl J Med 1995, 333:1581-7.

5. Roger VL, Go AS, Lloyd-Jones DM, Benjamin EJ, Berry JD, Borden WB, et al: Heart disease and stroke statistics-2012 update: a report from the American Heart Association. Circulation 2012, 125:e2-220.

6. Sacco RL, Shi T, Zamanillo MC, Kargman DE: Predictors of mortality and recurrence after hospitalized cerebral infarction in an urban community: the Northern Manhattan Stroke Study. Neurology 1994, 44:626-34.

7. Donnan GA, Fisher M, Macleod M, Davis SM: Stroke. Lancet 2008, 371:1612-23.

\section{P54}

Light sedation strategies and posttraumatic stress disorder: a systematic review and network meta-analysis

Antonio Paulo Nassar Junior ${ }^{*}$, Fernando G Zampieri, Otavio T Ranzani, Marcelo Park

Hospital das Clinicas, Faculty of Medicine, University of São Paulo, SP, Brazil Critical Care 2015, 19(Suppl 2):P54; doi:10.1186/cc14694
Introduction: Strategies aiming at light sedation are associated with decreased times on mechanical ventilation. However, awake or easily aroused patients may be prone to have greater prevalence of posttraumatic stress disorder. This systematic review and meta-analysis aimed to evaluate the safety of light sedation strategies in the prevalence of posttraumatic stress disorder.

Methods: We searched MEDLINE, Scopus and Web of Science from inception to November 2014 for randomized controlled trials which compared a light sedation strategy with a deeper sedation strategy and addressed posttraumatic stress disorder prevalence as a specific outcome.

Results: Five studies fulfilled our inclusion criteria and were included in the meta-analysis. Two studies compared daily sedation interruption with usual care (92 patients), two studies compared a light sedation protocol with daily sedation interruption (47 patients) and one study compared light and deep sedation (102 patients). Compared with usual sedation care/deep sedation, neither daily interruption of sedation (OR $=0.66,95 \% \mathrm{Cl} 0.22-1.98)$ nor a light sedation protocol $(\mathrm{OR}=0.90,95 \%$ $\mathrm{Cl}$ 0.27-3.05) were associated with increased risks on long-term PTSD prevalence. Heterogeneity was moderate $\left(l^{2}=40 \%\right)$.

Conclusion: Light sedation strategies seem to be safe in terms of posttraumatic stress disorder prevalence. However, the small number of included trials and patients may not be sufficient to drive strong statements. Ongoing large trials will be able to answer this question.

P55

Midterm survival and neurological outcome of mild therapeutic hypothermia after cardiac arrest in a community hospital in São Paulo, Brazil

Carlos Alberto C de Abreu Filho

Hospital Municipal Dr. Moysés Deutsch, Jardim Angela, São Paulo, SP, Brazil Critical Care 2015, 19(Suppl 2):P55; doi:10.1186/cc14695

Introduction: Mild therapeutic hypothermia (MTH) is a powerful therapy to improve survival and neurological outcome after cardiac arrest. It is technically simple and feasible to be implemented at the community level. Our objective is to analyze midterm survival and neurological outcome of patients submitted to MTH in a community hospital of a developing country.

Methods: Retrospective cohort study of patients treated with MTH after cardiac arrest in a community hospital in São Paulo, Brazil. After return of spontaneous circulation (ROSC), unconscious survivors of cardiac arrest were submitted to MTH, using topical ice and cold saline infusions, in order to cool patients to $32-34^{\circ} \mathrm{C}$, and to maintain the goal temperature for 24 hours. Esophageal temperature was monitored for all patients during the hypothermia and rewarming process $\left(1^{\circ} \mathrm{C}\right.$ each 8 hours). Continuous intravenous sedation analgesia was maintained for 48 hours. The Glasgow Outcome Scale (GOS) was used to analyze the neurological outcome at 30 days, and 1 year after hospital discharge; GOS $>3$ was considered a good neurological outcome.

Results: From January 2012 to February 2015, 148 patients were submitted to MTH after cardiac arrest; 109 patients $(73.6 \%)$ had out-ofhospital cardiac arrest and $39(26.4 \%)$ had intrahospital cardiac arrest. Mean age was $35.51 \pm 11.08$ years, 92 patients $(62.1 \%)$ were male. The mean causes of cardiac arrests were: exogenous intoxication (52.7 \%), acute myocardial infarction $(37.1 \%)$ and acute respiratory failure (10.2\%). Initial rhythm was ventricular fibrillation/pulseless tachycardia $(47.9 \%)$, asystole $(29.8 \%)$ and pulseless electrical activity (22.3\%). The mean time of cardiorespiratory resuscitation was $36.4 \pm 17.6$ minutes, the mean time from ROSC to initiation of MTH was $167.54 \pm 59.1$ minutes, and the mean time from initiation of therapeutic hypothermia to goal temperature was $151.81 \pm 75.4$ minutes. Mean complications during MTH were: pneumonia (38.5\%), cardiac arrhythmias (31.7\%) and coagulopathy (11.4\%). Hospital mortality (30 days) was $18.9 \%$; among the survivors, $72.5 \%$ of the patients had GOS $>3$ at hospital discharge. One-year survival was $70.9 \%$, among the patients who survive more than 1 year after hospital discharge, $74.5 \%$ had GOS $>3$.

Conclusion: MTH is a safe and effective therapy to improve midterm survival and neurological outcome after cardiac arrest in a community hospital. 
P56

Influence of the duration of brain death protocol on losses of potential donors by cardiac arrest

Miriam Cristine V Machado*, Artur Montemezzo, Fernanda Cani, Gabriel Torres, Glauco A Westphal, Joel de Andrade, Leandro S Botelho, Tiago A Slaviero, Tiago C Carnin

Central de Notificação, Captação e Distribuição de Órgãos do Estado de Santa Catarina (CNCDO/SC), Florianópolis, SC, Brazil

Critical Care 2015, 19(Suppl 2):P56; doi:10.1186/cc14696

Introduction: Organ transplantation is, in many cases, the only therapeutic option for patients with terminal organ dysfunction. Organ donation from deceased donors in brain death (BD) is the main source of organs for transplants. Goal-oriented protocols are associated with reduction of cardiac arrests in potential donors. However, there is not much information about chronological goals and the influence of the length of donor management on the loss of donors by cardiac arrest. The severe inflammatory reaction that occurs from the BD installation tends to amplify over time and could contribute to cardiovascular deterioration and occurrence of cardiac arrest.

Objective: To evaluate the influence of the duration of brain death protocol (DBDP) on losses of potential donors by cardiac arrest.

Methods: Retrospective analysis of potential donors notified to the Transplantation Center of the State of Santa Catarina in the period from May 2012 to April 2013. We determined the odds ratio of losses by cardiac arrest according to DBDP to identify the chronological threshold at which the probability of losses due to cardiac arrest increases. $p$ values $<0.05$ were considered significant.

Results: A total of 384 potential donors were analyzed and 272 were effective donors. There was a greater chance of cardiac arrests after the 30-hour threshold (OR 1.67, $95 \% \mathrm{Cl}: 0.38-0.93)$ and the lowest risk of stops was identified in the range of $12-30$ hours (OR 0.32, $95 \% \mathrm{Cl}: 0.19$ 0.52). Multivariate analysis identified the following variables associated with cardiac arrest: potential donor management outside the ICU ( $p=$ 0.001 ), adherence to management checklist, DBDP up to 30 hours ( $p=$ 0.003 ) and DBDP between 12 and 30 hours $(p=0.004)$. Protocols lasting more than 30 hours had a higher percentage of cardiac arrests $(35.4 \%$ vs. $24.6 \%, p=0.022$ ), and longer time intervals to perform the second clinical test $(14: 51 \pm 13: 02$ vs. 9:46 \pm 4:20, and $35: 37 \pm 29: 43$ vs. $11: 36 \pm$ 7:55, $p<0.001)$.

Conclusion: The long duration between the first clinical test for diagnosis of $\mathrm{BD}$ and the harvesting of organs may be an important risk factor for the occurrence of cardiac arrest in deceased organ donors.

\section{P58}

Analysis of influenza and parainfluenza respiratory infection in a private tertiary hospital in São Paulo, Brazil

Frederico P Lomar*, Carmen SV Barbas, Gustavo FJ de Matos, Humberto B Bogossian, Telma Anunes

Hospital Israelita Albert Einstein, Morumbi, São Paulo, SP, Brazil Critical Care 2015, 19(Suppl 2):P58; doi:10.1186/cc14697

Introduction: There are different viruses that cause respiratory infections. Most of the time it is very difficult to know the causative agent from the respiratory symptoms. In order to identify the possibility of a virus being the causative agent of the respiratory infection and to identify the type of virus that is causing the respiratory symptoms, nasal swabs were collected from patients with symptoms of acute respiratory infection.

Methods: During a 6-month period (1 February-31 July 2014) 365 nasal swabs were collected from patients with symptomatic respiratory infections admitted to Hospital Israelita Albert Einstein, São Paulo, Brazil for polymerase chain reaction virus detection (CLART Pneumovir) capable of detecting 14 respiratory viruses.

Results: Almost half of the samples collected tested positive. Specifically, $48.76 \%(178 / 365)$ of samples were positive for respiratory virus. Syncycial respiratory virus accounted for $19.66 \%$ (35/178), influenza H3N2 for $14.61 \%$ (26/178), parainfluenza virus for $7.3 \%(13 / 178)$, influenza B virus for $6.18 \%$ (11/178), influenza H1N1 for $1.69 \%$ (3/178) and influenza C for $1.69 \%(3 / 178)$.
Conclusion: Respiratory virus is a common cause of respiratory infection requiring hospital admission, rhinovirus being the most prevalent. The identification of the type of respiratory virosis is crucial for virus isolation precautions.

P59

Analysis of the functional diagnosis and Tobin index for failure in weaning from mechanical ventilation Maíra J Maturana*, Fabiana RF Arnone, Gabriela M Lucin, Larissa Domanski, Luiz Alberto M Knaut, Tais G de Matos, Esperidião E Aquim 1-Faculdade Inspirar, Cutiriba-PR, São Francisco, Curitiba, SP, Brazil Critical Care 2015, 19(Suppl 2):P59; doi:10.1186/cc14698

Introduction: There is a big divergence regarding the indexes that show whether an extubation process is successful or not. Regardless of this, the spontaneous breathing trial is the most recommended for that aim.

Objective: To identify the reason for failure in the weaning process and to relate it to the Tobin index.

Methods: Experimental study of patients admitted to the ICUs of the Tabalhador, Vita Curitiba, Vita Batel Hospitals and the Neurology Institute of Curitiba (INC), in the city of Curitiba, between April and December 2014. Thirty spontaneous breathing trials were taken with pressure support (PS) of 7, positive end-expiratory pressure (PEEP) of $5 \mathrm{cmH}_{2} \mathrm{O}$ and inspired oxygen fraction $=0.4$ for 30 minutes in 17 men and 13 women, with an average age of $51.4( \pm 24.2)$, who have been intubated for more than 24 hours in mechanical ventilation and are able to go through the mechanical ventilation weaning process. Upon failure of the SBT, the reason for the lack of success should be classified through functional diagnosis, divided into increase of resistance, characterized by decrease in respiratory rate (FR35), decrease of chest expansibility, pulmonary auscultation with minor vesicular groan diminished or ceased, noises from crackling or small bubbling and usage of accessory inspiratory muscle formation or still alteration of functional reserve. After the identification of the functional diagnosis, he Tobin index was calculated with zero PS and PEEP maintained at $5 \mathrm{cmH}_{2} \mathrm{O}$.

Results: Of the 30 TER 14 evolved with failure, where FR was predictive of failure in the weaning process and when related to the Tobin index (Figure 1). Increased FR (Figure 1A) showed a slightly positive correlation $\left(r_{\mathrm{pb}}=0.367 ; p=0.005\right)$, whilst decreased FR (Figure 1B) showed a slightly negative correlation $\left(r_{\mathrm{pb}}=-0.554 ; p=0.000\right)$.

Conclusion: The functional diagnosis may be an auxiliary predictive indicator for the failure in mechanical ventilation weaning when related to the Tobin index.

\section{References}

1. Faustino EA: Mecânica Pulmonar de Pacientes em Suporte Ventilatório na Unidade de Terapia Intensiva. Conceitos e Monitorização. Rev Bras Ter Intensiva 2007, 19:161-169.

2. Goldwasser R, et al: III Consenso Brasileiro de Ventilação Mecânica: Desmame e Interrupção da Ventilação Mecânica. J Bras Pneumol São Paulo 2007, 33:128-36.

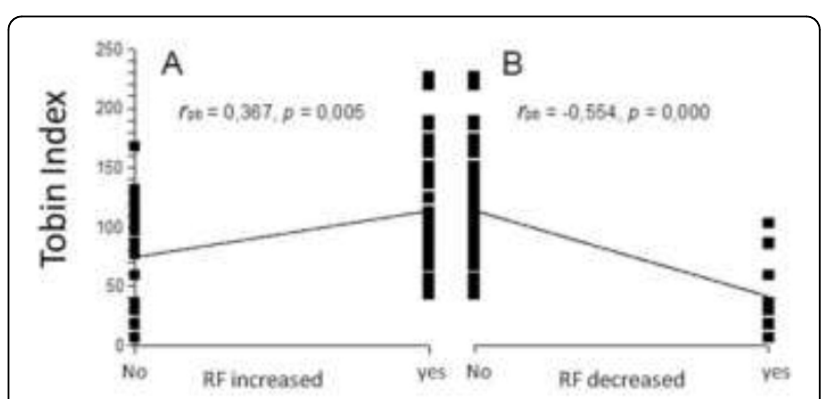

Figure 1(abstract cc14698) A Relationship between the Tobin index and the increase of respiratory frequency (RF). B Relationship between the Tobin index and the reduction of respiratory frequency (RF) 
P61

Comparing CDC's surveillance definitions and CPIS score in diagnosing ventilator-associated pneumonia: an observational study

Álvaro Koenig*, Dimitri S Possamai, Fernanda P de Aguiar, Glauco A Westphal, Kenia Fujiwara, Lucas R Ramos, Milton C Filho, Miriam Cristine Machado, Renata Waltrick, Valmir João de S Filho

Hospital Municipal São José e Centro Hospitalar Unimed, Joinville, SP, Brazil Critical Care 2015, 19(Suppl 2):P61; doi:10.1186/cc14699

Introduction: The National Healthcare Safety Network/Center for Disease Control and Prevention (NHSN/CDC) published in 2013 a new surveillance protocol in order to standardize the ventilator-associated pneumonia (VAP) confirmation criteria and, consequently, to increase the reliability of indicators in different institutions.

Objective: To evaluate the degree of agreement of CDC's new surveillance definitions and clinical criteria, using the CPIS score, in diagnosing VAP.

Methods: From August 2013 to June 2014 all patients on mechanical ventilation for longer than 48 hours in two critical care units in a public and a private general hospital were included in the study. On a daily basis, ventilated patients were evaluated by respiratory physiotherapists using the CPIS score and, independently, by the infection preventionist nurse using CDC's new surveillance definitions. CPIS score $=7$ was considered a clinical diagnosis, and, when associated with a semiquantitative culture with $10^{4}$ colony-forming units, as a definitive diagnosis of VAP.

Results: Eight hundred and one patients were admitted to both ICUs during the study period. One hundred and sixty-eight were on mechanical ventilation for more than 48 hours. Thirty-eight patients were diagnosed with pneumonia using the clinical criteria (13.8/1000 patients/ day on ventilation). Eighteen of these patients were diagnosed with infectious conditions associated with mechanical ventilation (IVAC) and 14 of them had a diagnosis of probable VAP (5.23/1000 patients/day on ventilation). Compared with clinical criteria, the $C D C^{\prime}$ s surveillance definitions had sensitivity $=0.37$, specificity $=1.0$, positive predictive value $=1.0$ and negative predictive value $=0.84$.

Conclusion: Compared with clinical criteria, using the CPIS score, the CDC's new surveillance definitions had a low sensitivity and may not be appropriate as a surveillance method.

P62

\section{Is unplanned extubation avoidable?}

Corinne Taniguchi*, Carolina SA Azevedo, Cilene Saghabi, Erica A Giovanetti, Guilherme PP de Schettino, Gustavo C da Ferreira, Gustavo FJ de Matos, Karina T Timenetsky, Raquel AC Eid, Ricardo Stus

Hospital Israelita Albert Einstein, Morumbi, São Paulo, SP, Brazil

Critical Care 2015, 19(Suppl 2):P62; doi:10.1186/cc14700

Introduction: Unplanned extubation (UE) is usually associated with a longer duration of mechanical ventilation (MV), ICU stay and hospitalization. In the Albert Einstein Hospital, it was noticed that there had been an increase in the number of UEs at the beginning of 2014 when protocols such as the daily sedation interruptions were established. UE is more common in patients who are agitated, when they have low levels of sedation or when the endotracheal tube is not well secured.

Objective: To evaluate an UE prevention program based on five actions. Methods: The UE ratio from April to August 2014 was compared with that of the same period from the previous year, comparing the UE index before and after the adoption of a prevention program for patients with a high risk of UE. The prevention of UE was based on five elements: the risk patients were identified during the patient's daily discussion; the endotracheal tube being secured by two different means; arm restraints; the sedation protocol being properly applied; and a sign indicating the potential risk was placed beside the bed.

Results: During the period April-August 2013 there was a total of 1793 patients on MV against 1720 patients in 2014. During this period, there were 12 UEs in 2013 and eight in 2014 generating an index of 0.7 and 0.5 respectively. In 2014, after the prevention program, there were $33 \%$ less UEs. In 2013 the majority of the UEs occurred with patients who were aware of the intubation and their surroundings $(n=8)$. Within 12 patients, four had UEs owing to poor tube security. Five of those 12 patients were sedated, only one was agitated and two were in the process of spontaneous trials. In point of fact, these two patients did not need any ventilatory assistance after extubation; six of the patients were reintubated and five had to use NIV. In 2014 the majority of the UEs also occurred in patients who were aware of the intubation and their surroundings ( $n=7$ ) and only one patient had improper tube security. They were all identified as being at a high risk of UE, all restrained, and with double tube security. Three of them were sedated, and were being ventilated in controlled mode. The other five patients were breathing spontaneously (PSV). As the necessity for ventilator intervention after extubation, four of the eight were reintubated; three needed NIV and one needed no support.

Conclusion: Although this analysis was carried out over a short period of time, the program and the effort of the staff was invaluable in order to diminish and control the number of UEs in our ICU, resulting in a level lower than $3 \%$.

Acknowledgements: The authors thank all ICU staff for their efforts and for engaging in this project with their ideas and actions

P63

Mechanical ventilation: does the moment of initial use or length of stay on mechanical ventilation have any relation to severity of illness and outcome?

Marina Parente Albuquerque*, Eduardo Queiroz da Cunha, Carlos Augusto Ramos Feijó, Allison Emidio Pinheiro Pereira Borges,

Natália Linhares Ponte Aragão, Túlio Sugette de Aguiar,

Francisco Albano de Meneses

Centro de Terapia Intensiva, Hospital Geral de Fortaleza, Fortaleza, CE, Brasil Critical Care 2015, 19(Suppl 2):P63; doi:10.1186/cc14701

Introduction: In the modern ICU, technologies are able to keep patients alive for prolonged periods of time, even despite ongoing life-threatening illness. Mechanical ventilation (MV) is crucial in most cases, although invasive $\mathrm{MV}$, when prolonged, may be associated with increased morbidity and mortality.

Objective: To verify the severity of illness in ICU patients, at admission, and its relation to invasive MV. To identify whether length of stay on MV or the moment of support implementation (at admission or during the ICU stay) relates to severity of illness and in-hospital/ICU outcomes.

Methods: Retrospective study, with clinical and surgical adult patients, admitted to the General Hospital of Fortaleza's ICU, from November 2014 to February 2015. Patients were divided into two groups: G1, patients on $\mathrm{MV}$; and G2, nonventilated patients. We analyzed the length of stay on MV (stratified into ranges of 1-7, 8-21 and >21 days) and its relation with disease severity, length of ICU stay and outcomes. Descriptive statistical analysis was used for demographic characters, $t$ test for evaluation of continuous variables, chi-square test for categorical variables and ANOVA for multiple comparisons, all with SPSS software. Patients with incomplete data were excluded from the analysis.

Results: We studied 86 patients, $51.16 \%$ were men, age average was $53.95 \pm 19.99$ years, average APACHE II score was $14.48 \pm 7.21$, average admission SOFA score was $4.92 \pm 4.01$ and average ICU stay period was $13.52 \pm 12.88$ days. In G1, $62.8 \%$ of patients $(n=61(70.9 \%))$ had invasive MV when admitted to the ICU. G1 patients had higher severity of illness scores: APACHE II 17.18 vs. 8.85 , admission SOFA 6.29 vs. 2.15 $(p<0.001)$. Despite stratification of time on MV, we observed no significant difference between the severity scores at admission. ICU and in-hospital mortality was higher in G1 (41.4\% vs. $3.6 \%, p=0.001$, and 52.9 vs. 14.8 $\mathrm{p}=0.002$, respectively). After the eighth day of MV, the average ICU stay increased in parallel with the duration of $M C(p<0.001)$. It's relevant to mention that $18.03 £$ of G1 used MV for more than 2 days. The moment of MC support initiation did not show statistically significant association with severity scores, length of ICU stay, or hospital/ICU mortality.

Conclusion: MV use was related to severity of illness, length of ICU stay, and in-hospital/ICU mortality. ICU length of stay increased with the duration of MV, from the second week forward. The moment of MV support initial use, however, did not seem relevant. 
P64

Monitoring the patient under mechanical ventilation: a Brazilian survey Alexandre M Isola*, Eliana B Caser, Ricardo G Rodrigues, Carmen SV Barbas Grupo de Assistência Ventilatória, Universidade de São Paulo, AvBrooklyn Novo, São Paulo, SP, Brazil

Critical Care 2015, 19(Suppl 2):P64; doi:10.1186/cc14702

Introduction: Adequate monitoring of the patient on mechanical ventilation (IMV) reduces complications and improves safety. In order to know more about this important issue in Brazil, we performed an electronic survey.

Methods: An open web-based electronic survey with questions about mechanical ventilation became available for intensive care practitioners in Brazil (DATAVENT) from 17 November 2013 to 31 August 2014 regarding the following questions: 1 . Which of these predictive indexes or maneuvers do you (or someone in your ICU staff) routinely apply during the IMV withdrawal process? 2. Check what of these parameters are measured or calculated in your ICU daily practice? 3. How do you obtain the driving pressure value? 4. How do you obtain the resistive pressure value? 5. Check what are the imaging resources available in your hospital for the patient under IMV? 6. What is the value of tidal volume $(\mathrm{ml} / \mathrm{kg}$ of predicted body weight (PBW)) that you use in patients under IMV without lung disease, including immediate postoperative period?

Results: There were 418 responders. Two hundred and ninety-seven of the intensive care practitioners $(71.05 \%)$ use $\mathrm{SpO}_{2}$ as an index in withdrawal process. Two hundred and seventy-four $(65.55 \%)$ use the rapid shallow breathing index. One hundred and five $(25.12 \%)$ use the maximal inspiratory pressure (PiMax) and 32 (7.66\%) use the integrative weaning index (IWI). In total, $336(80.38 \%)$ measure plateau pressure, 295 $(70.57 \%)$ measure intrinsic PEEP, 243 (58.13\%) measure static compliance, 99 (23.68\%) measure driving pressure and 98 (23.44\%) measure resistive pressure as routine. Thirty-eight $(9.09 \%)$ have access to monitor work of breath (WOB). A total of 216 (51.67\%) does not know how to obtain the driving pressure. A total of $241(57.65 \%)$ answered correctly how to obtain resistive pressure. A total of $329(78.71 \%)$ has access to conventional computerized tomography in their hospitals. A total of 245 (58.61 \%) has ultrasound and 223 (53.35\%) have echocardiography available. A total of 246 (58.85\%) perform chest radiography on a daily basis. A total of 183 (43.77\%) keeps the tidal volume of a patient under IMV without lung diseases (including postoperative patients) in $8 \mathrm{ml} / \mathrm{kg} / \mathrm{PBW}$. In total, $139(33.25 \%)$ use $6 \mathrm{ml} /$ kg/PBW, 68 (16.26 \%) use 7 ml/kg/PBW, 14 (3.35\%) use 9-10 ml/kg/PBW and 14 (3.35\%) use less than $6 \mathrm{ml} / \mathrm{kg} / \mathrm{PBW}$.

Conclusion: Monitoring of the patient under IMV is realized, focusing on the withdrawing process and basic mechanical parameters. Practitioners need to know more about obtaining the driving pressure and its important safety value, aiming to improve its use in their daily practice. Image resources availability on the ICU can be improved, mainly echography resources. The use of a tidal volume of $6 \mathrm{ml} / \mathrm{kg} / \mathrm{PBW}$ in patients without lung disease should be encouraged.

P65

Predictive factors of noninvasive ventilation failure in a pediatric ICU

Marcela B Alith ${ }^{1,2^{*}}$, Lilian AY Fernandes ${ }^{1}$, Rodrigo C Borges ${ }^{1}$

Regina HA Quinzani', Dumara N Oliveira', Andréia M Ferreira'

Nilce C Oliveira', Alexandra S Colombo

${ }^{1}$ University Hospital, University of São Paulo, Brazil; ${ }^{2}$ Federal University of São Paulo, Paulista School of Medicine (UNIFESP/EPM), Brazil

Critical Care 2015, 19(Suppl 2):P65; doi:10.1186/cc14703

Introduction: Noninvasive ventilation (NIV) has been developed to reduce complications associated with tracheal intubation and conventional mechanical ventilation. The aim of NIV is to gain control of acute respiratory failure, avoiding intubation; however, when intubation is required, its application should not be delayed, as this may result in a worse prognosis. This is the main reason to look for reliable failure signs of the technique.

Objective: To identify failure prognostic signs of NIV in pediatric acute respiratory failure.
Methods: This retrospective study was based on data collection from medical records of patients admitted to the pediatric ICU of University Hospital, University of São Paulo, from March to September 2012 and during the same period in 2013. Patients were divided into two groups: success group, in which patients who used NIV did not require intubation; and failure group, which included all patients who required intubation. The following variables were analyzed: age, sex, weight, personal history, previous use of oxygen, NIV success or failure, cause of failure, respiratory rate, heart rate, oxygen saturation, PRISM, PIM, the NIV devices and ventilatory parameters during NIV placement and during withdrawal.

Results: The charts of 112 patients were analyzed, 55 in the success group and 57 in the failure group. Most children who failed $(32.14 \%)$ were male. The median PRISM value in the failure group was 7 (5-8) ( $p=$ $0.000)$ and the median time of NIV use in this group was $570(182-1230)$ minutes $(p=0.000)$. In the univariate analysis, PEEP $(p=0.003)$, fraction of inspired oxygen $(p=0.000)$, oxygen saturation $(p=0.014)$, respiratory rate $(p=0.000)$, heart rate $(p=0.000)$ and need for sedation $(p=0.000)$ had a statistically significant difference in the moment of NIV withdrawal in the failure group. Logistic regression analysis showed that the independent factors significantly related to NIV failure were PRISM, total time in minutes and respiratory rate at the moment of withdrawal, considering the statistically significant value of $p<0.05$.

Conclusion: The PRISM value, NIV duration and respiratory rate can predict NIV failure in the pediatric population.

P70

Use of a hygroscopic heat-and-moisture exchanger during the rapid shallow breathing index

Rodrigo C Borges*, Leda TY Silveira, Juliana B Fernandes, Natalia S Arco, Samira P Furtado, Alexandra S Colombo

Hospital Universitário da Universidade de São Paulo, SP, Brasil

Critical Care 2015, 19(Suppl 2):P70; doi:10.1186/cc14704

Introduction: Heat-and-moisture exchangers (HMEs) have been increasingly used to heat and humidify the inspired gases in patients undergoing mechanical ventilation. However, little is known about its interference during measurement of the rapid shallow breathing index (RSBI).

Objective: The objective of this study was to evaluate the effect of the use of a hygroscopic HME during the measurement of RSBI in patients under mechanical ventilation (MV).

Methods: Randomized and controlled clinical study in patients admitted to the ICU. Inclusion criteria were patients of age $\geq 18$ years, with MV for at least 24 hours through an orotracheal tube (OTT) with an internal diameter of 7.5-9.0 mm, who were in the process of MV weaning. Patients were randomly allocated into the HME group or the non-HME group by a nonrelated investigator. Before the RSBI, subjects received respiratory physical therapy (bronchial hygiene therapy and tracheal suctioning) and were ventilated at pressure support ventilation with PEEP $=5-8 \mathrm{cmH}_{2} \mathrm{O}$, pressure support $=7-12 \mathrm{cmH}_{2} \mathrm{O}, \mathrm{FiO}_{2}<40 \%$, aiming peripheral $\mathrm{O}_{2}$ saturation $>90 \%$, tidal volume between 6 and $8 \mathrm{ml} / \mathrm{kg}$ and respiratory frequency $<30 \mathrm{bpm}$ for 30 minutes. Soon after, minute ventilation, dynamic compliance, airway resistance and respiratory frequency were registered by $\mathrm{MV}$. The bed head was elevated at $45^{\circ}$, subjects ventilated with $\mathrm{FiO}_{2}=100 \%$ for 1 minute, then disconnected from the ventilator, and the RSBI was then measured with a spirometer connected to the patient's OTT. For the HME group, a hygroscopic HME (Bact-HME; Pharma Systems, Knivsta, Sweden) was placed between the spirometer and the patient's OTT. Other collected data were clinical history (comorbidities, diagnosis, motive of admission), antropometric data, SAPS III and Sepsisrelated Organ Failure Assessment (SOFA) at the first 24 hours in the ICU, MV duration, ICU length of stay, presence of sepsis, use of corticoids, vasoactive drugs and dialysis days, and laboratorial examinations.

Result: Twenty-six subjects were assessed: 14 in the HME group and 12 in the non-HME group. Groups were not different regarding clinical conditions (comorbidities, cause of intubation, SAPS III, SOFA, MV duration, MV parameters, respiratory parameters, dose of sedation up to the moment of the RSBI measurement). The non-HME group presented a higher vasoactive drug dose $(p=0.04)$. No statistical significance was 
found between the measured RSBI for the HME group and the non-HME group.

Conclusion: Use of a hygroscopic HME filter does not interfere with RSBI measurement and could be an alternative to avoid spirometer contamination.

P71

An electronic warning system helps to reduce the time to diagnosis of sepsis

Miriam Cristine V Machado*, Álvaro Koenig, Geonice Sperotto,

Glauco A Westphal

Centro Hospitalar Unimed, Joinville, SP, Brazil

Critical Care 2015, 19(Suppl 2):P71; doi:10.1186/cc14705

Introduction: Reducing the time for the diagnosis of sepsis is a critical component to reduce the mortality rate related to severe sepsis and septic shock. The use of electronic warning devices may help in speeding the identification of sepsis risk patients.

Objective: To evaluate the effect of an electronic alert system for the identification of septic patients on the time to antibiotic administration and mortality.

Methods: An observational study that analyzed 480 patients with severe sepsis and septic shock in the period 2010-2014. An automatic warning system was implemented in 2010 to allow the identification of patients at risk for sepsis. This system sent automatic e-alerts for nurses of the wards through mobile devices. The Wilcoxon test and chi-square test were used for data analysis. $p<0.05$ was considered significant.

Results: After implementation of the automated system, the time between screening and diagnosis decreased over the 4-year period (2010: 3:30 hours, 2011: 1:50 hours, 2012: 1:26 hours, 2013: 1:18 hours, 2014: $1: 31$ hours, $p=0.02$ ). The total time between screening and antibiotic administration also reduced (2010: 5:36 hours, 2011: 3:00 hours, 2012: 2:30 hours, 2013: 3:03 hours, and 2014: 3:28 hours, $p<0.02$ ), with a concomitant mortality rate reduction (2010: $38.1 \%, 2011: 29.3 \%$, 2012: $25.3 \%, 2013: 19.6 \%$ and 2014: $24.1 \%, p=0.03)$. Comparing survivors and nonsurvivors, we observed that the speed in screening patients at risk was similar (survivors: 2:22 \pm 4:32 vs. nonsurvivors: 2:29 \pm 5:06 p.m., $p=0.82$ ). The variable "antibiotics in less than 1 hour" did not differ between survivors and nonsurvivors (161/350; $46 \%$ vs. $55 / 130 ; 42.3 \%$, $p=0.47)$. However, nonsurvivors were older $(69.5 \pm 15$ years vs. $55.7 \pm$ 21.2 years, $p<0.001$ ), more sick (APACHE II score: $27.0 \pm 8.1$ vs. $19 \pm 9.2$ $p<0.001)$ and showed higher levels of lactate at diagnosis $(3.3 \pm 2.9$ $\mathrm{mmol} / \mathrm{l}$ vs. $2.4 \pm 1.7 \mathrm{mmol} / \mathrm{l}, p<0.01)$. At the end of 6 hours the lactate level was similar (survivors: $3.1 \pm 3.8 \mathrm{mmol} / \mathrm{l}$ vs. non-survivors: $0.4 \pm 1.9$ $\mathrm{mmol} / \mathrm{l}, p=0.09$ ).

Conclusion: The electronic warning system helped to reduce the time necessary to perform the diagnosis of sepsis and the time to antibiotics. There was an association among the decrease in the time to diagnosis and mortality reduction. With the reduction in sepsis diagnosis time, intrinsic variables of patients seem to gain more weight in the risk of death associated with severe sepsis and septic shock.

P72

Blood lactate levels and/or norepinephrine requirements for risk stratification in sepsis

Diego O Cortés*, Arthur Cezar M Xavier, Bruno R de Almeida, Érica C de Vieira - Jacques Creteur, Jean-Louis Vincent, João Cláudio Lyra, Sylmara Zandona Department of Intensive Care, Hôpital Erasme, Anderlecht, Brussels, Belgium Critical Care 2015, 19(Suppl 2):P72; doi:10.1186/cc14706

Introduction: Recent large multicenter studies on early resuscitation protocols for sepsis in the emergency room (ER) have shown a mortality rate of $19 \%$ in the control groups $[1,2]$. These results suggest that the strategies used to include patients in these studies (high lactate or use of norepinephrine) did not identify a population at high risk of mortality. We explored the prognostic values of these criteria in an ICU population.

Methods: All admissions to our department of intensive care in 2013 were retrospectively screened to identify patients who had an initial elevated lactate ( $\geq 2 \mathrm{mEq} / \mathrm{l})$ or needed norepinephrine infusion (group OR) vs. those who had an initial elevated lactate and needed norepinephrine infusion (group AND) during the first 24 hours. We then classified the groups by the presence of sepsis at admission or not. The analysis was repeated using a lactate threshold of $\geq 4 \mathrm{mEq} / \mathrm{l}$. We collected relevant demographic and clinical data including the type of admission, data

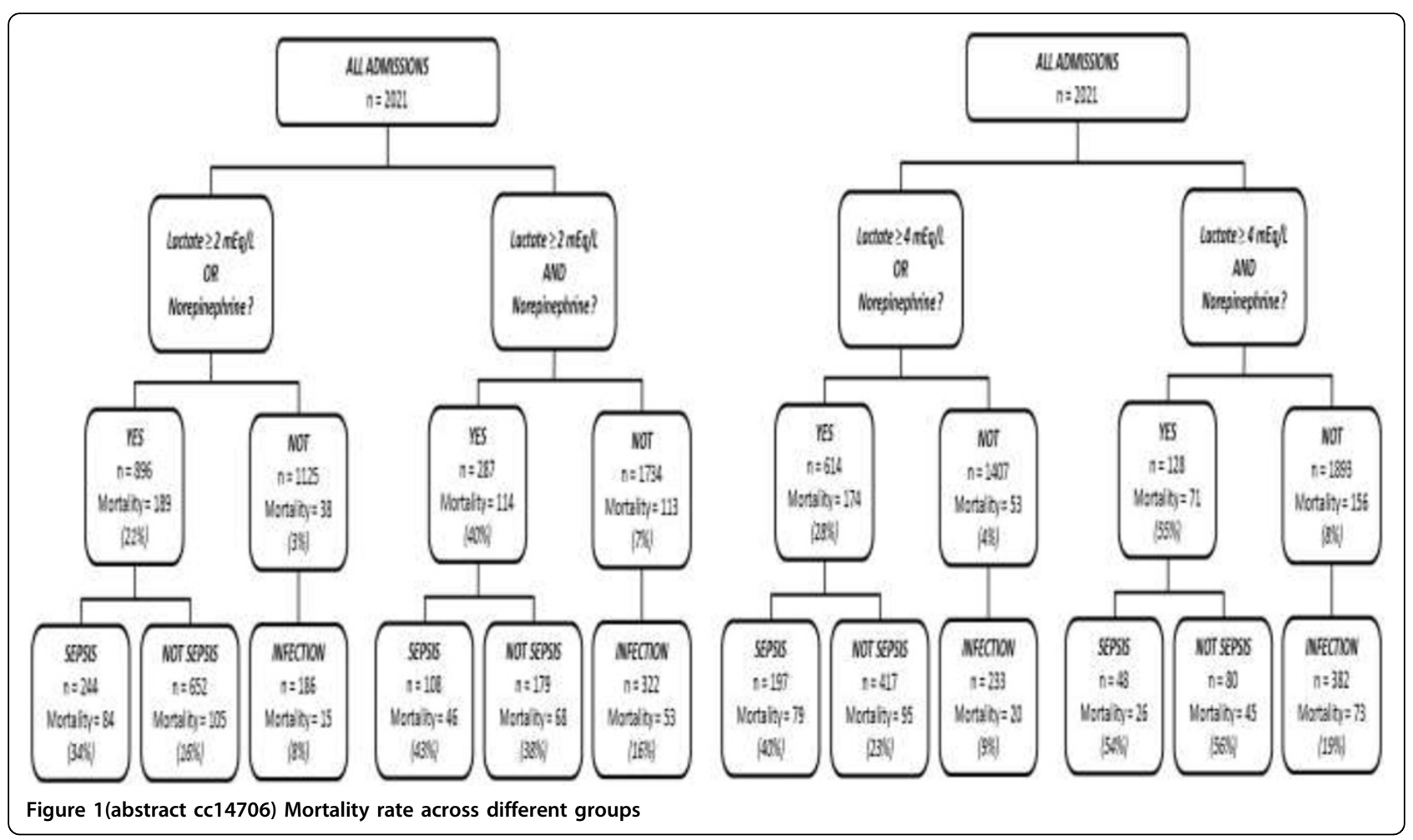


needed to calculate the sequential organ failure assessment (SOFA) score, and ICU mortality. All values are presented as proportions or median values (percentiles 25-75).

Results: Of the 2021 patients included, $430 \mathrm{had}$ a diagnosis of sepsis at admission; these patients had an ICU mortality of $23 \%$. One hundred and eighty-six needed norepinephrine during the first 24 hours and had a mortality rate of $40 \%, 166$ had an initial lactate $\geq 2 \mathrm{mEq} / \mathrm{l}$ with a mortality rate of $34 \%$, and 59 had an initial lactate $\geq 4 \mathrm{mEq} / \mathrm{l}$ with a mortality rate of $53 \%$. Using a lactate threshold of $\geq 2 \mathrm{mEq} / \mathrm{l}$, patients in the group AND needed higher norepinephrine doses (first 24 hours) $(0.39(0.16-0.76)$ vs. $0.14(0.03-0.44) \mu \mathrm{g} / \mathrm{kg} / \mathrm{minute})$, and $\geq 4 \mathrm{mEq} / \mathrm{l}$ yielded similar results. The mortality rates are shown in Figure 1. Patients in the group AND had higher mortality rates than the group OR, but there was a much smaller number of patients. Patients admitted with an infection but not fulfilling the criteria for the group AND or group OR had a lower mortality rate. Conclusion: Mortality in our septic population was higher than that reported in recent randomized controlled trials for early sepsis resuscitation in the ER $[1,2]$, limiting the external validity of these trial results to other ICU populations. Mortality was higher when hyperlactatemia and need for norepinephrine were present simultaneously compared with the presence of only one of these two criteria.

References

1. ProCESS trial. N Engl J Med 2014, 370:1683-93.

2. ARISE trial. N Engl J Med 2014, 371:1496-506.

\section{P74}

In the ICU and post ICU, platelets have more impact as a prognosis marker than leukocytes!

Carlos Augusto R Feijó*, Allison EPP Borges, Eduardo Q da Cunha,

Francisco A de Meneses, Marina P Albuquerque, Natália LP Aragão,

Tamara O Pinheiro, Túlio S de Aguiar

General Hospital of Fortaleza, Papicu, Fortaleza, CE, Brazil

Critical Care 2015, 19(Suppl 2):P74; doi:10.1186/cc14707

Introduction: In everyday practice, intensivists constantly search for tools to infer the prognosis of patients. The most simple and feasible markers stand out before the most complex and expensive ones. Traditionally, the white blood cell (WBC) count has been used as an inflammatory discriminant, rather than the platelet count. A comparison between these two parameters as predictors of ICU patient outcome, however, has been recently emphasized.

Objective: To correlate the levels of leukocytes, platelets, and the platelets/leukocytes ([P/L]) ratio with patient outcome, as far as discharge/ death in the ICU or in-hospital, concerning patients admitted to the ICU.

Methods: Data from patients admitted to the General Hospital of Fortaleza's ICU, from November 2014 to February 2015, were retrospectively analyzed. Complete blood count (CBC) data analyzed were collected on the first (D1) and fifth (D5) ICU days. Statistical analysis included the $t$ test for evaluation of the WBC count, platelet count and $[\mathrm{P} / \mathrm{L}]$ ratio relative to patient outcome, meaning ICU and in-hospital discharge/death. To compare these contents, the area under the ROC curve was computed for each index on D1 and D5. Patients with incomplete data were excluded from the study.

Results: From a total of 86 patients, $51 \%$ were male, mean age was $53 \pm$ 19 years and mean APACHE II score was $14 \pm 7$ points. CBC assessment demonstrated that the mean platelet number was significantly lower between patients who died and those who were discharged from the ICU and hospital, both on D1 and D5 (platelet count performed well in discriminating the ICU (AUC $=0.831$ ) and hospital ( $A U C=0.81$ ) outcome). Despite the poor performance on D1, the $D 5[\mathrm{P} / \mathrm{L}]$ ratio had regular performance for hospital outcome (AUC $=0.752$ ), and good for ICU outcome (AUC $=0.803$ ).

Conclusion: These findings suggest that continued assessment of $C B C$, especially platelets and the $[\mathrm{P} / \mathrm{L}]$ ratio, has better performance than leukocytes to infer the outcomes of the ICU and, subsequently, the hospital.
P75

Influence of mean arterial pressure and cardiac output on renal vascular tone reflected by the renal Doppler resistive index in critically ill patients

Raphael Augusto G de Oliveira*, Leandro U Taniguchi, Marcelo Park, Pedro $V$ Mendes

Hospital das Clínicas, Universidade de São Paulo, SP, Brazil

Critical Care 2015, 19(Suppl 2):P75; doi:10.1186/cc14708

Introduction: The renal Doppler resistive index (RI) is a non-invasive tool used to predict acute kidney injury (AKI) and evaluate renal vascular tone in the ICU setting. However, the real impact of hemodynamic parameter variations on $\mathrm{Rl}$ in critically ill patients is unknown.

Objective: To evaluate the influence of mean arterial pressure (MAP) and cardiac output (CO) on RI in critically ill patients.

Methods: Prospective observational study performed in the medicalsurgical ICU from August 2014 to December 2014. RI was performed daily until ICU discharge, death or need for renal replacement therapy (RRT). Transthoracic echocardiography was performed immediately after RI analysis to estimate cardiac output using the velocity-time integral (VTI) at the left ventricular outflow tract. All clinical and laboratorial data were obtained routinely during daily ultrasound examinations. Patients with chronic renal disease or on dialysis were excluded. Transient AKI was defined by normalization of renal function within 48 hours of AKI onset. Persistent AKI was defined by nonresolution of AKI within 48 hours of onset or need for RRT.

Results: Twenty-six patients were included $(61 \%$ medical admissions, $77 \%$ male, SAPS 3 of $52 \pm 14$ ). Seventy-three percent of patients developed AKI during the ICU stay (35\% had persistent AKI and $15 \%$ required RRT). Patients with persistent AKI had higher values of serum creatinine (1.76 \pm $0.82 \mathrm{mg} / \mathrm{dl}$ compared with $1.06 \pm 0.35 \mathrm{mg} / \mathrm{dl}$ in the transient AKI group or $0.79 \pm 0.29 \mathrm{mg} / \mathrm{dl}$ in the without AKI group, $p<0.01$ between groups) and RI $(0.74 \pm 0.07$ compared with $0.65 \pm 0.08$ in the transient AKI group or $0.65 \pm$ 0.05 in the without AKI group, $p<0.01$ between groups). There was no difference between mean MAP $(88 \pm 15 \mathrm{mmHg})$ in patients without AKI compared with patients with AKI $(84 \pm 13 \mathrm{mmHg}, p=0.429)$ and between

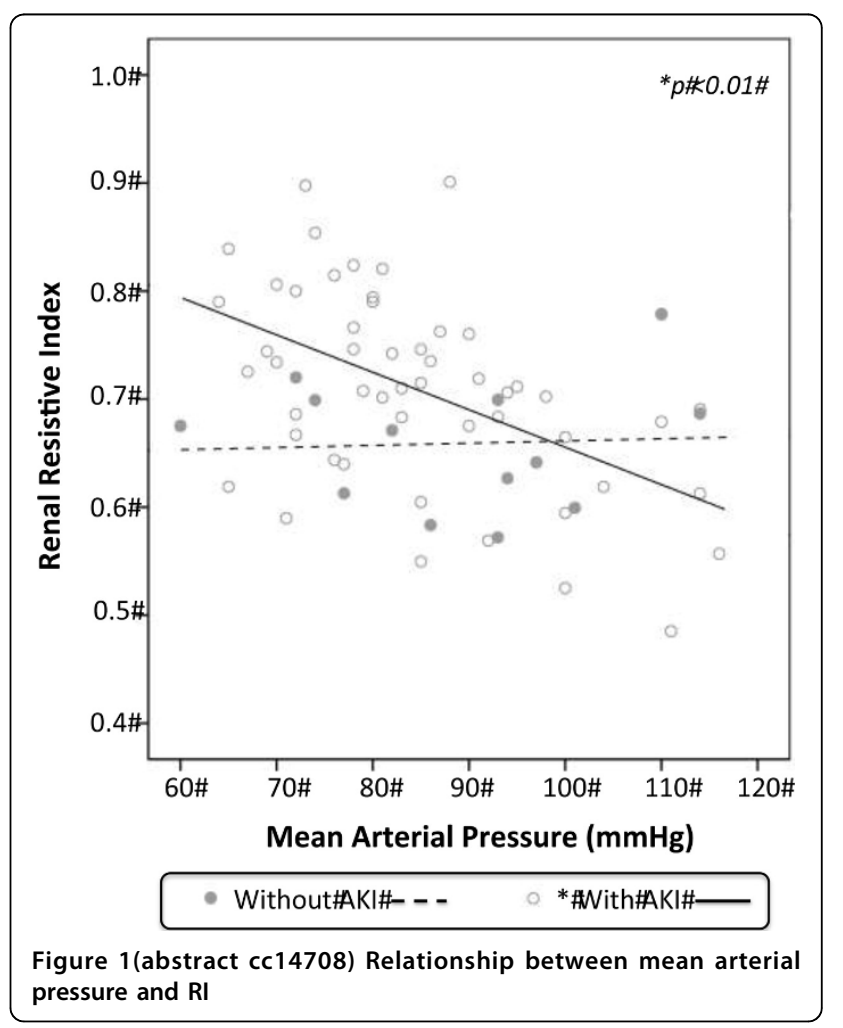


mean CO $(7.35 \pm 1.53 \mathrm{l} /$ minute) in patients without AKI compared with patients with AKI $(7.44 \pm 1.70 \mathrm{l} /$ minute, $p=0.848)$. MAP and RI demonstrated a negative correlation $(r=-0.507, p<0.01$; Figure 1$)$ in patients with AKI $(r=-0.455, p<0.05$ in transient AKI and $r=-0.372, p<0.05$ in persistent AKI) and no correlation ( $r=0.053, p=0.864)$ in those who did not develop AKI. There was no correlation between $\mathrm{CO}$ and $\mathrm{RI}$ in patients with AKI ( $p=0.532)$ or without AKI $(p=0.59)$.

Conclusion: We observed a negative correlation between RI and MAP in patients with AKI. No correlation was observed between $\mathrm{Rl}$ and $\mathrm{CO}$ in the different groups.

\section{P76}

ICU admissions and outcomes during the 2014 FIFA World Cup

Décio Diament", Andreia Pardini, Felipe M de T Piza,

Guilherme de PP Schettino, Thiago D Corrêa, Flavia R Citadin

Adult Intensive Care Unit, Hospital Israelita Albert Einstein, Morumbi, São

Paulo, SP, Brazil

Critical Care 2015, 19(Suppl 2):P76; doi:10.1186/cc14709

Introduction: There are some data suggesting that stressful events, such as soccer games, could affect the incidence and mortality due to cardiovascular disease. However, data on the impact of large-scale international events on the pattern of ICU admissions and outcomes of critically ill patients are limited.

Objective: To address the profile and outcomes of the ICU admissions in a private, high-complexity hospital during the 2014 FIFA World Cup held in Brazil, compared with the same period in the previous year.

Methods: Cross-sectional observational study. All adult patients admitted to a 41-bed medical-surgical ICU of a tertiary care private hospital in São Paulo, Brazil from 12 June to 13 July 2013 (control period) and from 12 June to 13 July 2014 (FIFA World Cup period) were included in this study. Demographic data, SAPS 3 score, clinical and outcome data were retrieved from an electronic ICU quality registry (Epimed Monitor System). Comparisons were performed between the World Cup and the control periods.

Results: Two hundred and sixty-seven patients were admitted to the ICU during the control period and 251 patients during the World Cup period. The proportion of male patients did not differ between the two periods ( $58 \%$ vs. $54 \%$, respectively for control and World Cup periods, $p=0.37$ ), as well as the proportion of clinical, elective and emergency surgery admissions $(p=0.18)$. Patients admitted to the ICU during the World Cup period were slightly younger (mean (SD)) than patients admitted during the control period (63 years $( \pm 18)$ vs. 67 years $( \pm 18), p=0.031$ ) and had lower SAPS 3 score $(45.3( \pm 15.9)$ vs. $49.5( \pm 18.5), p=0.006)$. The ICU mortality rate was $6.8 \%(17 / 251)$ for the World Cup period and $6.7 \%$ $(18 / 267)$ for the control period (adjusted OR, 1.90; $95 \% \mathrm{Cl}, 0.84-4.30$; $p=0.13$ ). While the median (IQR) length of ICU stay did not differ between the World Cup and control periods (2 (1 to 4 ) days vs. 2 (1 to 4$)$, respectively, $p=0.75)$, the length of hospital stay was significantly lower during the World Cup period (11 (5 to 28 ) days vs. 14 (7 to 32 ) days, $p=0.01$ ).

Conclusion: Although patients admitted to the ICU of a private hospital during the World Cup were slightly younger and less sick compared with patients admitted during the same period in the previous year, the pattern of ICU admissions and the outcomes were not affected. Our results should be compared with those obtained in the other 11 cities selected for the tournament, including private and public hospitals.

\section{P77}

Neither too much, neither too little! Positive fluid balance and ICU outcome

Allison EPP Borges*, Carlos Augusto R Feijó, Eduardo Q da Cunha, Francisco A de Meneses, Marina P Albuquerque, Natália LP Aragão,

Tamara O Pinheiro, Túlio S de Aguiar

General Hospital of Fortaleza, Papicu, Fortaleza, SP, Brazil

Critical Care 2015, 19(Suppl 2):P77; doi:10.1186/cc14710

Introduction: The fluid balance of critically ill patients has emerged as a potential marker of disease severity. This is associated with worse outcome and prolonged time of use of intensive care support in the ICU.
Objective: To research the influence of positive fluid balance in the first 72 hours of hospitalization in the ICU on organ dysfunction and outcome. Methods: Retrospective study including patients admitted to the General Hospital of Fortaleza/SESA ICU, from November 2014 to February 2015. Patients were characterized by the presence of circulatory and renal dysfunction at the discretion of the SOFA score. The days of mechanical ventilation were computed. The multivariate analysis was performed by ANOVA test.

Results: From a total of 86 patients, $51 \%$ were men, the mean age was $53.95 \pm 19.99$ years, and mean APACHE II score was $14.47 \pm 7.2$ points. Of these, 68 patients $(79 \%)$ had a fluid balance measured in the first 72 hours of admission and were included in the study. The fluid balance was higher in clinical patients, rather than surgical patients (4183.86 vs. $2491.88 \mathrm{ml} ; p=0.049$ ). Patients who did not use mechanical ventilation had lower values of positive fluid balance compared with those who used that support (1687.47 vs. $4499.5 \mathrm{ml}, p=0.02$ ). There was a more meaningful fluid overload in patients with renal and cardiovascular dysfunctions than in those without these disorders ((4317.59 vs. 3465.61 $\mathrm{ml} ; p=0.09$ ); (3184.95 vs. $4405.23 \mathrm{ml} ; p=0.092$ ), respectively). The length of stay in the ICU for those patients who had fluid balance greater than $2000 \mathrm{ml}$ in the first 72 hours was 16.06 days, while for those with fluid balance $<2000 \mathrm{ml}$ was 9.1 days $(p=0.041)$. There were differences between the fluid balance values of patients who died and those who were discharged from the ICU, but without statistical significance.

Conclusion: Our findings show that fluid overload with positive cumulative fluid balance during the first 72 hours in the ICU is associated with longer ICU length of stay, but without impact on ICU mortality.

\section{P78}

Patterns of organic dysfunction in severe sepsis and septic shock patients admitted to the ICU from the emergency department: a 4-year retrospective cohort

Alan Felipe Sakai", Leonardo Lima Rocha, Camila Menezes Souza Pessoa, Thiago Domingos Corrêa, Murillo Santucci César de Assunção

Intensive Care Unit, Hospital Israelita Albert Einstein, São Paulo, SP, Brazil Critical Care 2015, 19(Suppl 2):P78; doi:10.1186/cc14711

Introduction: Mortality rates for severe sepsis and septic shock are decreasing through the years worldwide. Most of this improvement in mortality is associated with protocols for early recognition, resuscitation and adequate initial antibiotic choice in patients presenting with sepsis to the emergency department.

Objective: To assess the pattern of organic dysfunction as described by the Sequential Organ Failure Assessment (SOFA) score in a 4-year period.

Methods: Retrospective cohort study that included all clinical patients admitted to the ICU from the emergency department with diagnosis of severe sepsis or septic shock. Surgical patients and patients admitted from the ward or referrals were excluded. Demographic data and SOFA score (including individual organic dysfunctions and the general score, which is the sum of organ-specific scores) were collected from January 2008 to December 2012 at admission, 48 hours and 7 days.

Results: We included a total of 472 patients. The median age was 70 (IQR $55-82$ ) and most patients were male (61.2\%). Respiratory tract infection was the most common infection site $(52.7 \%)$. The median APACHE II score was 19 (IQR 17-24) and the median SOFA score at admission was 4 (IQR 3-7). A total of $58.5 \%$ of the patients presented with severe sepsis. The SOFA score at admission was statistically significant throughout the years $(P=0.004)$. This was mainly due to circulatory and respiratory dysfunctions at admission, which significantly reduced throughout the years $(P<0.001$ and 0.045 , respectively). The SOFA score significantly decreased along the days (from admission to 7 days) $(P<0.0001)$ and this was consistent throughout the years.

Conclusion: Higher severity SOFA scores are decreasing through the years probably due to the increase in early recognition and resuscitation of severe sepsis and septic shock patients admitted from the emergency department. 
P81

Deleterious effects of severe sepsis and septic shock on physical activity in daily life, muscle strength and exercise capacity: a prospective cohort study

Rodrigo C Borges ${ }^{1 *}$, Celso RF Carvalho², Alexandra S Colombo', Mariucha PS Borges ${ }^{1}$, Francisco G Soriano ${ }^{1,3}$

${ }^{1}$ University Hospital, University of São Paulo, SP, Brazil; ${ }^{2}$ Physiotherapy Department, School of Medicine, University of São Paulo, SP, Brazil; ${ }^{3}$ Internal Medicine Department, School of Medicine, University of São Paulo, SP, Brazil Critical Care 2015, 19(Suppl 2):P81; doi:10.1186/cc14712

Introduction: Sepsis is a clinical problem of great relevance within the ICU because survivors can suffer from severe dysfunction and symptoms, such as fatigue, dyspnea, muscle weakness and a decrease in the healthrelated quality of life; however, the effects of this disease on physical activity in daily life in the short and medium term are not known.

Objective: The objective of the study was to quantify the physical activity in daily life, muscle strength and exercise capacity in the short and medium term in survivors from severe sepsis and septic shock. Furthermore, we investigated clinical and laboratory factors that determine muscle strength, exercise capacity and physical activity in daily life.

Methods: Prospective cohort study with a follow-up from hospital admission to 3 months after hospital discharge. Seventy-two patients admitted to the ICU due to severe sepsis or septic shock and a control group of healthy sedentary subjects $(n=50)$ were enrolled. All patients had their physical activity in daily life quantified by an accelerometer during their hospital stay and 3 months after hospital discharge. Exercise capacity (6-minute walking distance) and respiratory, handgrip and quadriceps muscle strength were also evaluated during hospitalization and 3 months after.

Results: During hospitalization, patients spent the majority of their time inactive in a lying or sitting position (90 $\pm 34 \%$ of daily time). Physical inactivity was partially reduced 3 months after hospital discharge $(58 \pm 20 \%$ of daily time). However, the time patients spent walking was only $63 \%$ of the time reported for healthy subjects. Patients also showed a reduction in walking intensity. At hospital discharge, muscle strength and exercise capacity were approximately $54 \%$ of the predicted value, and these parameters showed a small but significant increase in patients 3 months after hospital discharge (70\% of predicted value). A multivariate regression analysis demonstrated that the use of systemic corticosteroids and hospitalization time negatively influenced quadriceps strength and exercise capacity at the time of hospital discharge.

Conclusion: Our results strongly suggest that survivors of sepsis admitted to the ICU have a substantial reduction in physical activity, exercise capacity and muscle strength compared with healthy subjects that remains even 3 months after hospital discharge.

P84

Esophageal cancer in the ICU: a clinical-epidemiological retrospective study Fabrício RT de Carvalho*, Antônio Paulo Nassar, Lucas C Macedo, Pedro Caruso Unidade de Terapia Intensiva, Ac Camargo Cancer Center, SP, Brazil Critical Care 2015, 19(Suppl 2):P84; doi:10.1186/cc14713

Introduction: Esophageal cancer is a serious clinical condition with high mortality and morbidity, and during its evolution ICU admissions are common [1]. The main reasons for those admissions are elective postoperative conditions or clinical complications, such as bronchial aspiration owing to dysphagia, esophageal perforation and mediastinitis [2]. Despite the complexity of the disease, few data are available regarding esophageal cancer and critical care medicine.

Objective: To evaluate clinical and epidemiological characteristics of such a population during their ICU stay.

Methods: We performed a retrospective analysis of all cases with esophageal cancer that were admitted to the ICU of a large teaching hospital specializing in cancer in São Paulo, Brazil, between September 2009 and December 2014. Clinical and epidemiological characteristics of the patients were described, as well as risk factors identified regarding mortality during the ICU stay.

Results: A total of 228 patients were analyzed during the period. Mean age was $60 \pm 12$ years and $82.5 \%$ were male. Of the admissions, $50.4 \%$ were related to elective surgery monitoring, and $49.6 \%$ were clinical and emergency surgery admissions. The most frequent diagnosis that motivated
ICU admission was postoperative monitoring ( $56.1 \%)$, followed by acute respiratory failure $(11.4 \%)$ and severe sepsis $(7.5 \%)$. The mean value of SAPS 3 at admission was $59.3 \pm 15.3$. Overall mortality was $20.6 \%$ during the ICU stay; $12.8 \%$ were women and $87.2 \%$ men. Logistic regression identified that the only independent variable related with mortality was SAPS 3 at admission (OR: 0.03-0.14; $95 \% \mathrm{Cl}, p=0.003$ ).

Conclusion: Patients with esophageal cancer admitted to the ICU are predominantly men, admitted after a surgical procedure (elective or emergency). The only risk factor identified for ICU mortality was the value of SAPS 3 at admission. Other risk factors such as age, diagnosis of admission and organ dysfunction may be related to mortality but were not observed in our patients. Further studies regarding this issue, especially prospective studies, should be performed.

References

1. Lee L, Li C, Robert N, Latimer E, Carli F, Mulder DS, Fried GM, Ferri LE, Feldman LS: Economic impact of an enhanced recovery pathway for oesophagectomy. Br J Surg 2013, 100:1326-34

2. Bissell L, Khan OA, Mercer SJ, Somers SS, Toh SK: Long term outcomes following emergency intensive care readmission after elective oesophagectomy. Acta Chir Belg 2013, 113:14-8.

\section{P85}

Evolutive analysis of the SOFA score in critically ill massive burn patients during their stay in the ICU

Edvaldo V de Campos, Luciano Cesar P Azevedo, Marcelo Park

Hospital das Clínicas, University of São Paulo Medical School, São Paulo, SP, Brazil Critical Care 2015, 19(Suppl 2):P85; doi:10.1186/cc14714

Introduction: It is estimated that multiple organ dysfunction could be responsible for $80 \%$ of mortality in critically ill burn patients, mainly related to sepsis. However, there are no recommendations for monitoring organ dysfunction during the stay of burn patients in the ICU.

Objective: Our aim was to analyze and characterize organ dysfunction by the Sequential Organ Failure Assessment (SOFA) score in massive burn patients during their stay in the ICU with the hypothesis that survivors diverge from nonsurvivors during the length of stay in the ICU.

Methods: Retrospective cohort study employing data collected from May 2005 to April 2010 at an ICU specializing in burn patients at a teaching hospital. All patients admitted during this period were included. Data for physiological and epidemiological variables were collected at admission. During the ICU evaluation, the total SOFA score with its components were recorded from the first day to the seventh day. It was also recorded on the 14th, 21st and 28th days if the patient stayed in the ICU. The clinical outcomes collected were the ICU length of stay, hospital length of stay, and ICU and hospital mortality.

Results: One hundred and sixty-three consecutive patients were studied (male: $71 \%)$, with median age of $34(25,47)$ years and a hospital stay of 29 $(11,50)$ days. Incidence of inhalation injury was $45 \%$ and total burn surface area $(\%)$ was $29(18,43)$. The total SOFA score at admission in survivor patients was $1(1,4)$ and in nonsurvivors was $7(4,9)(P<0.05)$. Significant difference related to the total SOFA score was found during all other days analyzed. The analysis in the first 7 days with the receiver operating characteristic curve of worst individual organ dysfunction quantified through the total SOFA score and partitioned SOFA score showed good capacity to discriminate survivors and nonsurvivors between the respiratory, cardiovascular, renal and hematological components. Hepatic and neurological components did not present a good performance.

Conclusion: In our study, organ dysfunction quantified by total SOFA score and respiratory, cardiovascular, renal and hematological partitions was different between survivors and nonsurvivors during the ICU evolution.

P86

Factors for the development of pressure ulcers in patients with traumatic brain injury

Sibila L Osis*, Adriana M de Oliveira, Adriana R Marinho, Antonia OM Loureiro, Edilse S da Silva, Francisca das CF Carneiro, Juceny GU dos Santos, Solange Diccini

Instituto de Enfermeiros em Terapia Intensiva do Amazonas, Manaus, AM, Brazil

Critical Care 2015, 19(Suppl 2):P86; doi:10.1186/cc14715 
Table 1(abstract cc14715) Relation between average value of GCS, BS and pressure ulcers

\begin{tabular}{llll}
\hline Variable & \multicolumn{2}{l}{ Pressure ulcer } & $\boldsymbol{p}$ value \\
\cline { 2 - 3 } & Yes $(\boldsymbol{n}=\mathbf{4 5})$ & No $(\boldsymbol{n}=\mathbf{1 9 5})$ & \\
\hline Braden Scale & $10 \pm 1.4$ & $13.3 \pm 2.1$ & $<0.001^{*}$ \\
Glasgow Coma Scale & $9 \pm 2.5$ & $12 \pm 3$ & $<0.001^{*}$ \\
\hline
\end{tabular}

In patients with PU, 32 (71 \%) were admitted to the ICU (norepinephrine, $p<0.001$ ) *Student $t$ test

Introduction: Traumatic brain injury (TBI) is considered a public health problem by the World Health Organization because it is the major cause of sequelae among people younger than 44 years, affecting all races and ages [1]. The TBI patients are at risk for development of pressure ulcer (PU) due to the therapeutic used; hemodynamic and metabolic changes, immobility, loss of bladder and bowel control, changes in the ability of adequate nutritional intake and dependence on self-care are considered risk factors for development of PU $[2,3]$.

Objective: To evaluate the incidence of PU in patients with TBI and its relation to the level of consciousness and risk of PU development.

Methods: Prospective study in a referral hospital in neurotrauma in Manaus city, Amazonas State, in adult patients admitted with TBI from November 2013 to August 2014. We included patients aged 18 years or older that had a hospital stay of 24 hours or greater. The Glasgow Coma Scale (GCS) and the Braden Scale (BS) were applied. The study was approved by the institutional review board of the Universidade Federal de São Paulo, with the consent requirement obtained from the patient or family member. For statistical analysis we used the Epi $\operatorname{lnfo}^{\mathrm{TM}} 7$ program.

Results: A total of 240 patients with TBI was included in this analysis, of which 110 (45.5\%) Mild TBI, 69 (28.8\%) Moderate, and 61(25.4\%) Severe. The majority were male $(86.7 \%, n=208)$, with an average age of $35 \pm 12$ years, 209 (87.1\%) were not of light skin tone. For the BS only, seven (2.9\%) did not have risk for development of PU, all of TBI Mild. The incidence of PU occurred in $18.8 \%(n=45)$ of the patients, being three (6.6 \%) Mild TBI, 16 (35.5 \%) Moderate, and 26 (57.7 \%) Severe. Low score values on the GCS and BS were observed in patients who developed PU (Table 1).

Conclusion: There was a high incidence of PU, and patients with GCS and $\mathrm{BE}$ of low scores were more likely to develop the complication. Several factors increase the likelihood of PU in this population, so assessment and prevention measures must be strict at hospitalization.

References

1. Centers for Disease Control and Prevention: Surveillance for traumatic brain injury-related deaths-United States, 1997-2007. MMWR 2011, 60:1-32.

2. Cox J: Predictors of pressure ulcers in adult critical care patients. Am J Crit Care 2014, 20:364-75.

3. Wound, Ostomy and Continence Nurses Society: Guideline for prevention and management of pressure ulcers. Mount Laurel: WOCN: Ratliff CR, Tomaselli N 2010, 96.

\section{P87}

Improving postoperative outcomes in a Brazilian hospital through educational programs based on reports of an international database in cardiac surgery

Pedro Gabriel M de BE Silva*, Antonio Baruzzi, Denise Ramos,

Giuliano Generoso, Jose Teixeira, Marcelo Jamus, Mariana Okada, Nilza Lasta,

Thiago A Macedo, Valter Furlan

Hospital Totalcor, Cerqueira Cesar, São Paulo, SP, Brazil

Critical Care 2015, 19(Suppl 2):P87; doi:10.1186/cc14716

Introduction: Multicenter databases are useful tools for quality improvement programs. Most of this evidence is based on studies in North America and Europe and little is known in other regions. Since 2011, a Brazilian private cardiovascular center has joined an international registry of cardiac surgeries.
Table 1(abstract cc14716)

\begin{tabular}{|c|c|c|c|}
\hline CABG & $\begin{array}{l}2012 \\
(n=368)\end{array}$ & $\begin{array}{l}2013 \\
(n=299)\end{array}$ & $\begin{array}{l}p \\
\text { value }\end{array}$ \\
\hline $\begin{array}{l}\% \text { of CABGs using blood } \\
\text { transfusion }\end{array}$ & 48.7 & 37 & 0.005 \\
\hline Mean time for extubation (hours) & 11.3 & 4.3 & $<0.001$ \\
\hline ICU mean length of stay (hours) & 64.8 & 50.4 & 0.001 \\
\hline Mean PO length of stay (days) & 6.5 & 6 & NS \\
\hline $\begin{array}{l}\text { Hospital discharge on the fourth } \\
\text { PO day (\%) }\end{array}$ & 12.2 & 33.8 & $<0.001$ \\
\hline ICU readmission (\%) & 4.6 & 1.6 & 0.048 \\
\hline Hospital readmission <30 days (\%) & 13 & 4.7 & $<0.001$ \\
\hline 30-day mortality (\%) & 2.17 & $1 \%$ & 0.36 \\
\hline
\end{tabular}

Objective: To evaluate changes in quality indicators and clinical outcomes of cardiac surgery patients after a multifaceted educational program based on reports of an international database.

Methods: A multifaceted and continuous educational program based on trimestral reports from the international database was implemented in a Brazilian cardiovascular center. A local team targeted reductions in the time of mechanical ventilation (MV), in length of stay and in the number of inappropriate transfusions. A pilot protocol for rational use of blood products based on guidelines was developed in 2011 [1]. Standard criteria for sedation and extubation in the perioperative period were implemented in 2012. The best hospitals of the database were used as a benchmark to define goals with the surgical and clinical staff. All patients submitted to coronary artery bypass graft (CABG) surgeries were included in the analysis which compared pre and post program in order to observe the impact of the educational intervention.

Results: From January 2012 to December 2013, 667 CABGs were performed. The predicted risk of in-hospital mortality by the score of the Society of Thoracic Surgeons (validated in the hospital [2]) was $1.2 \%$ in 2012 and 0.96 $\%$ in 2013. As shown in Table 1, there was a reduction in transfusion comparing 2012 and 2013. The time in MV and the postoperative length of stay reduced in 2013. Mortality did not increase with an earlier extubation and discharge.

Conclusion: These results indicate that quality improvement program based on international database reports can improve outcomes in a Brazilian private hospital. Global registries can be useful tools to overcome gaps in clinical practice in different countries.

References

1. de Barros e Silva PGM, et al: Implantação de protocolo institucional para o uso racional de hemoderivados e seu impacto no pós-operatório de cirurgias de revascularização miocárdica. Einstein (São Paulo) 2013, 11:310-6.

2. Ikeoka DT, et al: Evaluation of the Society of Thoracic Surgeons score system for isolated coronary bypass graft surgery in a Brazilian population. Rev Bras Cir Cardiovasc 2014, 29:51-8.

P88

Meeting clinical goals for the maintenance of the potential organ donor can reduce the loss of donors by cardiac arrest

Miriam Cristine V Machado*, Artur Montemezzo, Fernanda Cani, Gabriel Torres

, Glauco A Westphal, Joel de Andrade, Leandro Botelho, Silvana Wagner, Stefan Halla, Tiago C Carnin

Central de Notificação, Captação e Distribuição de Órgãos do Estado de Santa Catarina, Florianópolis, SC, Brazil

Critical Care 2015, 19(Suppl 2):P88; doi:10.1186/cc14717

Introduction: The disproportion between the large organ demand and the low number of transplantations performed represents a serious public health problem worldwide. In Brazil, the loss of transplantable organs from deceased potential donors as a function of cardiac arrest is notably high. Objective: To test the hypothesis that a goal-directed protocol to guide the management of deceased donors may reduce the losses of potential donors due to cardiac arrest. 
Methods: Analysis of all deceased donors reported prospectively to CNCDO/SC, over six 4-month periods between May 2012 and April 2014. Hospitals were encouraged to use a checklist to obtain clinical goals during the management of deceased donors. The checklist was composed of the following goals: protocol duration $12-24$ hours, temperature $>35^{\circ} \mathrm{C}$, mean arterial pressure (MAP) $>65 \mathrm{mmHg}$, diuresis $1-4 \mathrm{ml} / \mathrm{kg} /$ hour, corticoids, vasopressin if MAP $<65 \mathrm{mmHg}$, tidal volume $6-8 \mathrm{ml} / \mathrm{kg}$, PEEP $8-10 \mathrm{cmH}_{2} \mathrm{O}$, sodium $<150 \mathrm{mEq} / \mathrm{l}$ and blood glucose $<180 \mathrm{mg} / \mathrm{dl}$. Effective donors were compared with losses by cardiac arrest. A logistic regression model was used to identify predictors of cardiac arrest with $p<0.05$.

Results: Of 771 consecutive deceased donors at 27 hospitals, 41 were excluded. Data from the remaining 730 were analyzed. There were 324 (42.0) effective donors, 145 (18.8\%) cardiac arrests, 226 family refusals and 35 contraindications. Compliance with the checklist increased from $52.1 \%$ in the first 4 -month period to $85.6 \%$ by the end of 2 years $(p<0.001)$. Cardiac arrests decreased from $19.8 \%$ (first 4-month period) to $14.6 \%$ (sixth 4 -month period) over 2 years $(p=0.26)$. Comparing the sixth 4 -month period with the period before the start of the study $(26.4 \%)$, there was statistical significance in the latter $(p=0.002)$, with maintenance of this performance in the two following 4-month periods (Quad 7: 13.8 \% and Quad 8: $12.1 \% ; p<0.001)$. Factors associated with cardiac arrest reduction were: use of the checklist (OR $0.27,95 \% \mathrm{Cl} 0.16-0.44, p<0.001)$ and temperature $>35^{\circ} \mathrm{C}(0.79,95 \% \mathrm{Cl} 0.19-0.79, p=0.006)$. The occurrence of cardiac arrests were inversely proportional to the number of interventions (no checklist: $56 \%, 0-1: 35 \%, 2: 46 \%, 3: 38.7 \%$, 4: $20.2 \%, 5: 17.4 \%, 6$ : $12 \%, 7: 11 \%)$. More than four interventions had less association with cardiac arrests (30.9 \% vs. $15.4 \%$, OR: $0.40,95 \% \mathrm{Cl} 0.24-0.69, p<0.001$ ). Conclusion: Meeting clinical goals during the management of deceased donors might reduce the loss of organ donors owing to cardiac arrest.

P89

Prognostic evaluation from different types of acidosis in high-risk surgical patients

João Manoel Silva Junior $r^{1,2,3^{*}}$, Amanda Maria RR de Oliveira ${ }^{1,2,3}$

Cristina P Amendola ${ }^{1,2,3}$, Fernando A Mendes ${ }^{1,2,3}$, LM Sá Malbouisson ${ }^{1,2,3}$, Maria José C Carmona ${ }^{1,2,3}$, Pedro MMV Nogueira ${ }^{1,2,3}$

${ }^{1}$ Anesthesiology Department, Hospital das Clinicas, São Paulo, SP, Brazil;

${ }^{2}$ Anesthesiology Department, Hospital do Servidor Público Estadual-IAMSPE, São Paulo, SP, Brazil; ${ }^{3}$ Intensive Care Department, Hospital do Câncer de Barretos, São Paulo, SP, Brazil

Critical Care 2015, 19(Suppl 2):P89; doi:10.1186/cc14718

Introduction: Acidosis is a very frequent disorder in surgical patients However, the nature of metabolic acidosis (hyperlactatemia, hyperchloremia, and others) seems to be indicative of worse clinical outcomes compared with $\mathrm{pH}$ value.

Objective: This study assessed the role of different types of acidosis in the outcome of high-risk surgeries.

Methods: Prospective, multicentric and observational study performed in three different tertiary hospitals. Patients who needed postoperative ICU admission were included in the study. Patients with low life expectancy

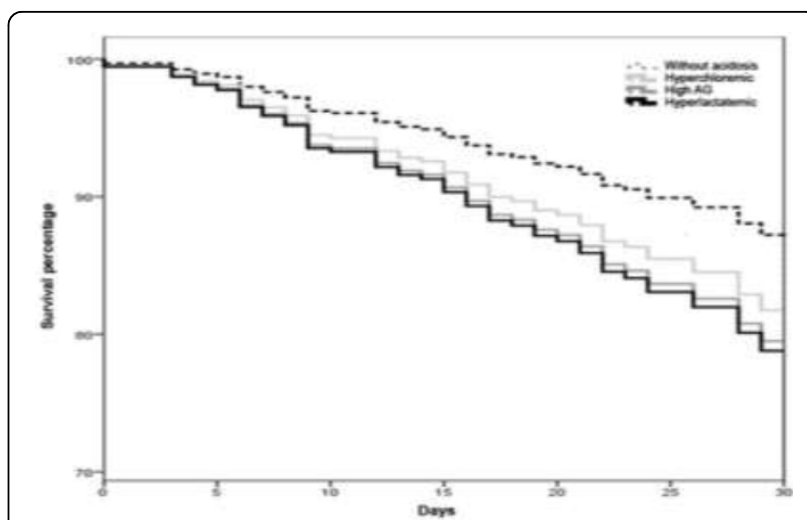

Figure 1 (abstract cc14718) Log rank test (Mantel-Cox, $\mathrm{p}=0.03$ )

(untreated cancer), hepatic failure, renal failure, and diabetes diagnosis were excluded. The patients were classified at ICU admission according to the presence and type of metabolic acidosis. The classification criteria were: base excess $<-4 \mathrm{mmol} / \mathrm{l}$; high albumin-corrected anion gap $>12 \mathrm{mmol} / \mathrm{l}$; and hyperlactatemia $>2 \mathrm{mmol} / \mathrm{l}$. Thus, acidosis was classified as hyperlactatemic, high or normal albumin-corrected anion gap (hyperchloremic).

Results: The study included 618 patients. Acidosis incidence at ICU admission was $59.1 \%$, being 148 (23.9\%) patients with hyperchloremia, 131 $(21.2 \%)$ with hyperlactatemia, 86 (13.9\%) with high anion gap, and 253 $(40.9 \%)$ without metabolic acidosis. Even though patients did not exhibit different demographic profile and severity, those who remained with acidosis after 12 hours, depending on group classification during the postoperative period, exhibited greater ICU complications: hyperlactatemia group $=68.8 \%$; high anion gap $=68.6 \%$; hyperchloremic $=65.8 \%$; and without acidosis $=59.3 \%, P=0.03$. Cardiovascular, neurologic, and renal dysfunctions were the main complications and the hyperlactatemia group exhibited the highest level. The same result was observed with respect to hospital mortality rate, $30.1 \%$ (HR 1.74, $95 \% \mathrm{Cl} 1.02-2.96$ ) hyperlactatemic; $24.3 \%$ (HR 1.68, $95 \% \mathrm{Cl} 0.85-3.81$ ) high anion gap; $18.4 \%$ (HR 1.47, $95 \% \mathrm{Cl}$ 0.75-2.89) hyperchloremic; and $10.3 \%$ no acidosis group (log-rank testMantel Cox, $P=0.03$ ). See Figure 1.

Conclusion: Metabolic acidosis in surgical patients is a highly prevalent postoperative complication, mainly related to hyperchloremia. Depending on the type, patients who developed metabolic acidosis postoperatively exhibited the worst outcomes compared with patients without acidosis, and the specific acidosis diagnosis can help in management.

Cite abstracts in this supplement using the relevant abstract number, e.g.: Silva Junior et al.: Prognostic evaluation from different types of acidosis in high-risk surgical patients. Critical Care 2015, 19(Suppl 2):P89 